ARTICLE

\title{
Coordination of endothelial cell positioning and fate specification by the epicardium
}

\author{
Pearl Quijada (1) 1,8, Michael A. Trembley', Adwiteeya Misra1,2, Jacquelyn A. Myers (10 3,4, \\ Cameron D. Baker (10 3,4, Marta Pérez-Hernández (10 ${ }^{5}$, Jason R. Myers ${ }^{3,4}$, Ronald A. Dirkx Jr. ${ }^{1}$, \\ Ethan David Cohen ${ }^{6}$, Mario Delmar ${ }^{5}$, John M. Ashton (i) ${ }^{3,4}$ \& Eric M. Small (1) ${ }^{1,2,7 \times}$
}

The organization of an integrated coronary vasculature requires the specification of immature endothelial cells (ECs) into arterial and venous fates based on their localization within the heart. It remains unclear how spatial information controls EC identity and behavior. Here we use single-cell RNA sequencing at key developmental timepoints to interrogate cellular contributions to coronary vessel patterning and maturation. We perform transcriptional profiling to define a heterogenous population of epicardium-derived cells (EPDCs) that express unique chemokine signatures. We identify a population of Slit2+ EPDCs that emerge following epithelial-tomesenchymal transition (EMT), which we term vascular guidepost cells. We show that the expression of guidepost-derived chemokines such as Slit2 are induced in epicardial cells undergoing EMT, while mesothelium-derived chemokines are silenced. We demonstrate that epicardium-specific deletion of myocardin-related transcription factors in mouse embryos disrupts the expression of key guidance cues and alters EPDC-EC signaling, leading to the persistence of an immature angiogenic EC identity and inappropriate accumulation of ECs on the epicardial surface. Our study suggests that EC pathfinding and fate specification is controlled by a common mechanism and guided by paracrine signaling from EPDCs linking epicardial EMT to EC localization and fate specification in the developing heart.

\footnotetext{
${ }^{1}$ Department of Medicine, Aab Cardiovascular Research Institute, University of Rochester School of Medicine and Dentistry, Rochester, NY, USA. ${ }^{2}$ Department of Biomedical Engineering, University of Rochester, Rochester, NY, USA. ${ }^{3}$ Genomics Research Center, University of Rochester School of Medicine and Dentistry, Rochester, NY, USA. ${ }^{4}$ Department of Microbiology and Immunology, University of Rochester School of Medicine and Dentistry, Rochester, NY, USA. ${ }^{5}$ Leon H. Charney Division of Cardiology, Department of Medicine, New York University School of Medicine, New York, NY, USA. ${ }^{6}$ Department of Pediatrics, University of Rochester School of Medicine and Dentistry, Rochester, NY, USA. 7 Department of Pharmacology and Physiology, University of Rochester, Rochester, NY, USA. ${ }^{8}$ Present address: Department of Integrative Biology and Physiology, Molecular Biology Institute, Eli \& Edythe Broad Center of Regenerative Medicine and Stem Cell Research, UCLA Cardiovascular Theme, David Geffen School of Medicine, University of California, Los Angeles, CA, USA. ${ }^{凶}$ email: eric_small@urmc.rochester.edu
} 
C oronary endothelial cells (ECs) organize into an intricate vascular network that provides the heart with oxygen and nutrients and removes metabolic waste. During embryonic development, localized specification of immature ECs into arterial and venous fates occurs within the compact myocardium and subepicardium, respectively ${ }^{1}$. EC fate specification and maturation are accomplished through cell-intrinsic transcriptional programs that facilitate appropriate interconnections of the blood-supplying and blood-draining vascular beds ${ }^{2}$. Analyses of intersomitic and retinal vessel development identified a population of endothelial tip cells that are particularly responsive to secreted angiogenic factors such as vascular endothelial growth factor (Vegf), highlighting the functional heterogeneity of ECs and revealing mechanisms governing vascular pathfinding, remodeling, and maturation ${ }^{3}$. Clinical studies aimed at improving perfusion of the ischemic heart and skeletal muscle have attempted to recapitulate these developmental angiogenic programs ${ }^{4}$. However, the mechanisms through which coronary ECs coordinate positional information and fate specification remain elusive, and current therapeutic strategies have failed to generate a robust, functional vascular network.

The epicardium consists of a single layer of mesothelial cells on the surface of the heart that harbors a population of multipotent progenitors. Following epithelial-to-mesenchymal transition (EMT), epicardium-derived cells (EPDCs) migrate into the compact myocardium and differentiate into cardiac fibroblast and vascular mural cell lineages ${ }^{5-7}$. Construction of the coronary plexus requires the integration of epicardium-derived mural cells with arterial and venous ECs derived from the sinus venosus and endocardium ${ }^{5,8,9}$. Genetic or mechanical disruption of the epicardium has also revealed important paracrine contributions to cardiomyocyte growth $^{10}$ and coronary plexus formation ${ }^{11,12}$. Our previous study found that epicardial EMT is required for coronary blood vessel maturation and integrity, at least partially via contributing vascular pericytes to the growing plexus ${ }^{7}$.

In this study, we performed single-cell RNA-sequencing of EPDCs and coronary ECs at critical developmental stages to gain insight into the mechanisms responsible for patterning of the developing coronary vasculature via distinct epicardial cell populations ${ }^{13-15}$. We found that epicardial EMT is not only responsible for the differentiation of EPDCs into vascular mural lineages ${ }^{7}$, but also restricts the expression of chemotactic signals to discrete populations of mural cells that provide detailed positional information, reminiscent of the guidepost neuron ${ }^{16}$. Genetic disruption of epicardial EMT in mice leads to profound alterations in EC developmental trajectory, which includes the accumulation of an immature EC population within the subepicardium. Importantly, EC maturation and migration are both directly controlled by angiogenic chemokines, providing a paradigm that coordinates EC localization and arteriovenous (AV) specification. Harnessing the principles that define the spatial architecture of the developing coronary vasculature may provide strategies to stimulate angiogenesis and improve perfusion of ischemic heart tissue, a limiting aspect of regenerative medicine approaches.

\section{Results}

Single-cell analysis of epicardium-derived cell heterogeneity. Coronary endothelial cell AV specification and integration of the arterial and venous vasculature coincides temporally with epicardial EMT, between embryonic day (E) 12.5 and E16.5 (Fig. 1a). To investigate epicardial contributions to the growing coronary plexus at these timepoints, GFP-positive $\left(\mathrm{GFP}^{+}\right)$ EPDCs were isolated from $\mathrm{Wt}^{\mathrm{CreERT} 2 /+}$; $\mathrm{Rosa}^{\mathrm{mTmG}}$ mouse embryos by fluorescence-activated cell sorting (FACS) (Fig. 1b, c and Supplementary Fig. 1a-d). GFP ${ }^{+}$cells displayed epicardial gene enrichment (Aldh1a2, Tbx18, Tcf21, Wt1) and did not express high levels of cardiomyocyte genes (Tnnt2, Myh7) (Supplementary Fig. 1e). Increased expression of the mesenchymal cell marker Pdgfra was observed in a number of $\mathrm{GFP}^{+}$cells at E16.5, consistent with the acquisition of a motile phenotype and differentiation into interstitial cell types (Supplementary Fig. 1f-h).

Single-cell RNA-sequencing (scRNA-seq) was performed on EPDCs captured using the $10 \times$ Genomics platform (Fig. 1d). We excluded cell doublets based upon unique molecular identifier counts, and mitochondrial and ribosomal gene expression patterns were analyzed and filtered to obtain 3405 (E12.5) and 2436 (E16.5) single EPDCs (Supplementary Fig. 2a, b). To define the cellular heterogeneity within the epicardium, we performed an integration of E12.5 and E16.5 data sets using canonical correlation analysis (CCA) followed by uniform manifold approximation and projection (UMAP) using Seurat to rule out batch effects (Supplementary Fig. 3a-d), and present a merged analysis of E12.5 and E16.5 cells (Fig. 1e). This analysis revealed 8 distinct populations ( $\mathrm{C} 1-\mathrm{C} 8)$ with a considerable contribution of proliferation state and developmental age to cellular phenotype (Fig. 1e, f and Supplementary Fig. 4a, b). To facilitate the identification of epicardial cell phenotypes, E12.5 and E16.5 data were merged and a CCA was performed with previously published scRNA-seq data obtained from the postnatal day 1 mouse heart ${ }^{17}$ (Supplementary Fig. 4c, d). Within these populations, five broad identities emerged, consistent with marker genes identified by hierarchical clustering and gene ontology (GO) analysis (Fig. 1g-k and Supplementary 5a, b; Supplementary Dataset 1): (1) early developmental stage progenitor (C1, C3); (2) early EMT (C5); (3) late developmental stage mesothelial (C2, C4); (4) late developmental stage EMT/ mesenchymal (C6, C7); and (5) a rare population of approximately 40 cells $(\mathrm{C} 8,1.64 \%$ of total) that display an enrichment in IGF pathway genes and express high levels of Cav1 previously implicated in zebrafish heart regeneration ${ }^{13}$.

EPDCs in C1 and C3 are characterized by robust expression of epicardial gene markers $T b x 18$ and $U p k 3 b^{18,19}$, and GO terms associated with proliferation and epithelial cell differentiation, defining populations of self-replicating E12.5 mesothelial cells (Fig. 1g, h). In contrast, C5 is enriched in E12.5 EPDCs that exhibit a predisposition towards EMT prior to the acquisition of a motile gene program, based on the expression of early markers of the mesenchymal and smooth muscle phenotype, including Pdgfra, Itgb5, Sox9, and Tagln2 20,21 (Fig. 1g, i). C2 and C4 contain an over-abundance of E16.5 EPDCs that express genes related to maintenance of mesothelial characteristics (Maf, Btg2), likely representing cells that remain on the cardiac surface 22,23 (Fig. 1g, j). Genes encoding extracellular matrix proteins such as Postn, Dpt, and Col3a1 are robustly expressed in E16.5 EPDCs in C6 and C7, consistent with the acquisition of a mesenchymal phenotype $^{20}$ (Fig. 1g, k). Of note, these data also revealed unique vascular programs based upon the emergence of angiogenesis among the most enriched GO terms at E16.5, and the enrichment of $H s p b 1$ and $D l k 1^{24,25}$ in mesothelial cells and Ramp2 and Sfrp2 26,27 in mesenchymal cells (Fig. 1g, j, k and Supplementary Fig. 5a).

Distinct epicardium-derived vascular guidance programs. We next evaluated the divergence of mesothelium and mesenchyme from a common epicardial progenitor, and the potential contribution of these distinct cellular populations to angiogenic processes, by assessing pseudotime trajectory using Monocle, an unsupervised learning algorithm that identifies branch points and cell commitment decisions ${ }^{28,29}$. We observed an abundance of E12.5 progenitor cells at the start of pseudotime (primarily $\mathrm{C} 1, \mathrm{C} 3$ ), that transition to 
a

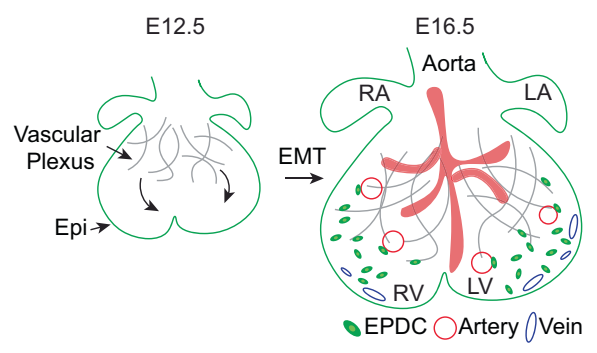

b

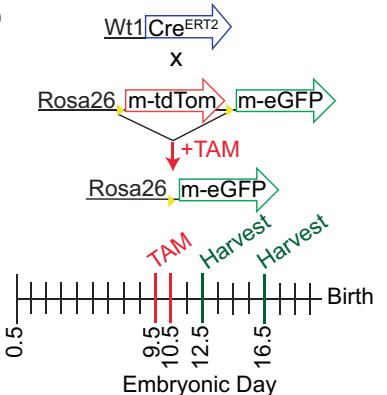

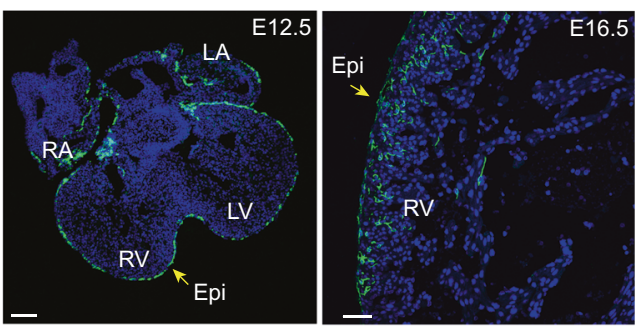

d

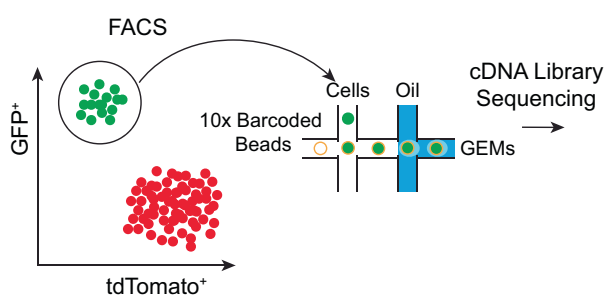

e

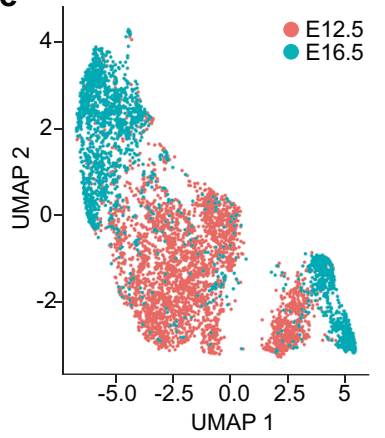

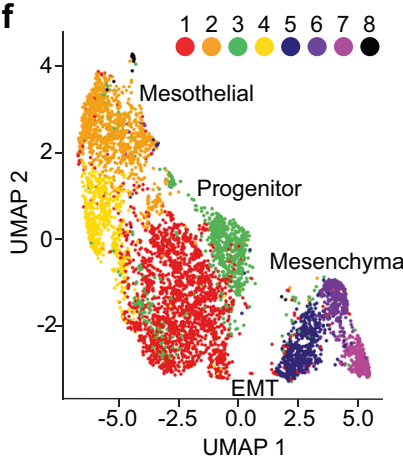

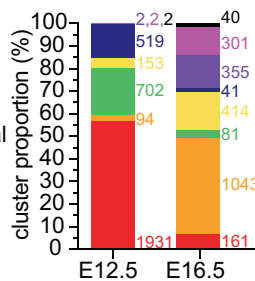

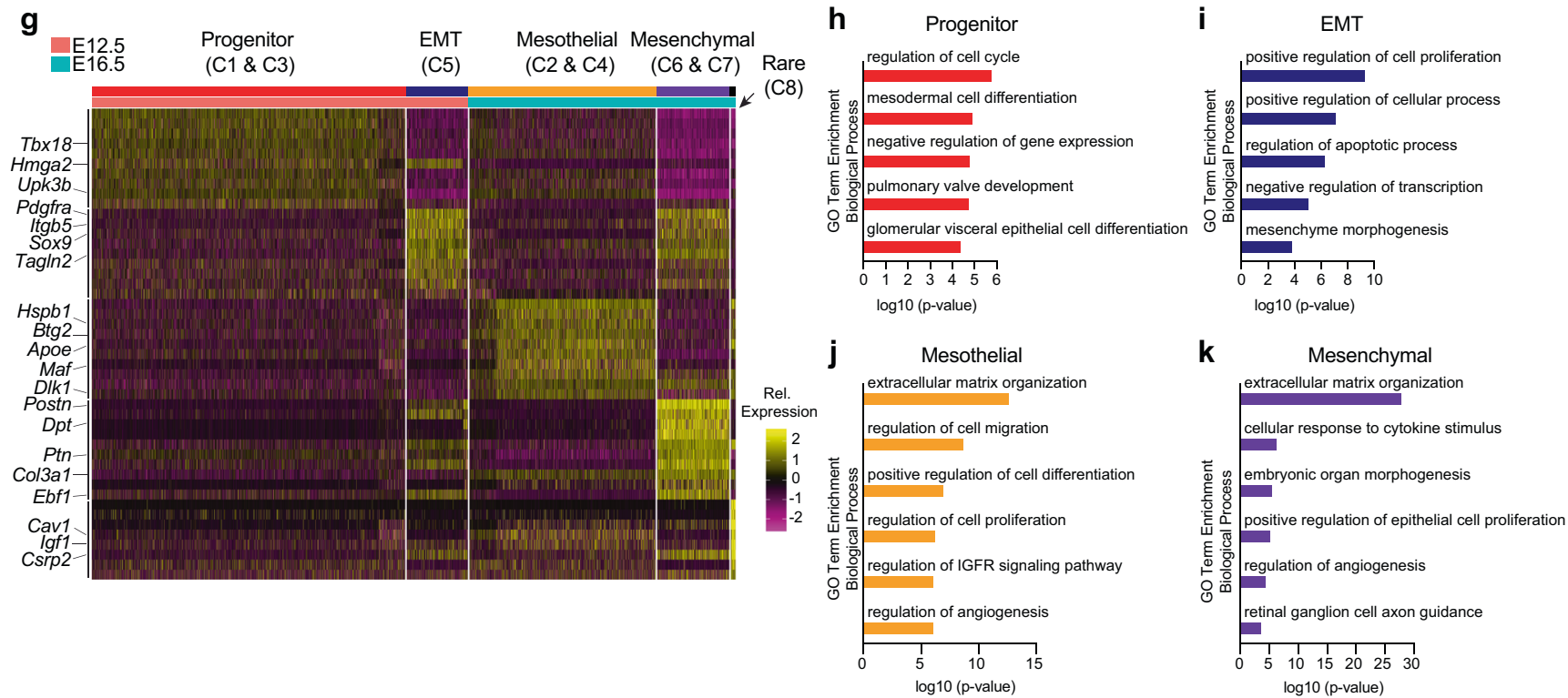

g

Fig. 1 Characterization of epicardial cell heterogeneity in the fetal heart. a Schematic of epicardium and vasculature development. $\mathbf{b}$ Experimental strategy for lineage tracing epicardium-derived cells in $\mathrm{Wt}_{1}$ CreERT2;R26 $\mathrm{mTmG}$ embryos. TAM was administered to pregnant dams at embryonic day (E) 9.5 and E10.5 and embryos were harvested at E12.5 and E16.5. c Representative images of mouse embryos at embryonic stage E12.5 and E16.5 used for collection of GFP+ epicardial cells (green). DAPI staining was utilized to visualize nuclei (blue). Scale bar is $100 \mu \mathrm{m}$ (E12.5) and 50 $\mu \mathrm{m}$ (E16.5). Immunostaining was repeated independently 3 times with similar results. d FACS-based enrichment of Wt7-lineage-derived epicardial cells prior to singlecell capture using 10× Genomics Chromium Controller. Refer to Supplementary Fig. 1c, d for FACS sequential gating and enrichment of epicardial cells. e and $\mathbf{f}$ UMAP of single-cell transcriptomes of E12.5 (3405 cells) and E16.5 (2436 cells) epicardial cells presented by e developmental stage and $\mathbf{f}$ cell identity, with the relative contribution of each cell cluster within each developmental stage indicated on the right. The number of cells per cluster (C) is listed next to the corresponding color in the graph. $\mathbf{g}$ Hierarchical clustering of differentially expressed genes by cell identity represented as a heat map. Relative (Rel.) expression represents scaled normalized expression. h-k GO analysis indicates most enriched biological processes within cells defined as $\mathbf{h}$ progenitor ( $\mathrm{C} 1$ and $\mathrm{C} 3$ ), i EMT (C5), j mesothelial (C2 and C4), and $\mathbf{k}$ mesenchymal (C6 and C7). GO-term enrichment significance was determined using Fisher exact test. Epi epicardium, EMT epithelial-to-mesenchymal transition, RA right atrium, RV right ventricle, LA left atrium, LV left ventricle, m-tdTom membrane tdTomato, m-eGFP membrane-enhanced green fluorescent protein, TAM tamoxifen, GEMs Gel Bead-in-Emulsion.

cell states ultimately dominated by E16.5 EPDCs (Fig. 2a, b and Supplementary Fig. 6a, b). Cell state 2 is enriched in E16.5 mesothelial cells $(\mathrm{C} 2, \mathrm{C} 4)$, while cell state 3 is composed of mesenchymal cells (C6, C7) following an early EMT intermediate (C5) (Fig. 2b). Analysis of pseudotime kinetics of differentially regulated genes across cell states revealed that mesothelial cells within state 2 maintain expression of genes such as $W t 1, M s l n$, and $U p k 3 b^{18}$, while cells in state 3 show downregulated expression $M s \ln$ and $U p k 3, b$ as well as $P d p n$ and $P d g f a^{30,31}$, markers that are generally associated with the mesothelium (Fig. $2 c$ ). Instead, state 3 cells are 
a

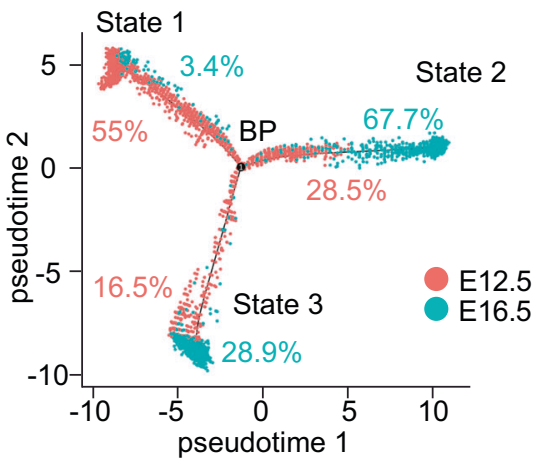

b

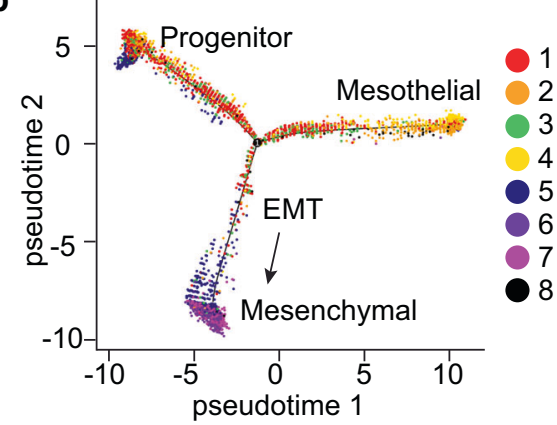

C

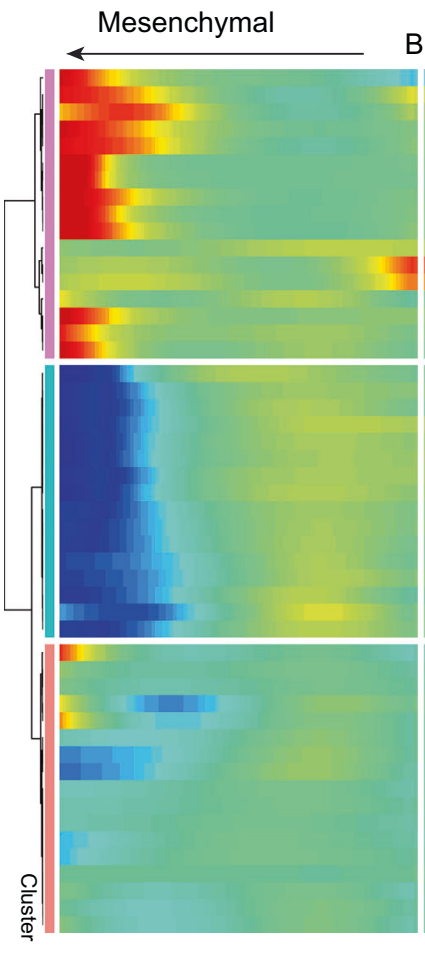

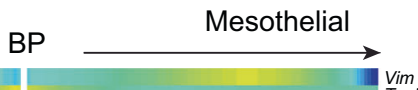

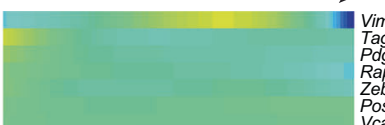

Tagln2
Pdgfra
Raph1
Zeb2 2 Postn
Vcam 1 Lhap
Serpine2 Emilin1 Mest
H2afz
Hagh 1 Hmgb1
$M d k$ Sept11
Glipr2 Glipr
Ptn Tnni1 Arhgap29 S100a6 Fbln2 Urrn 4 3 ib Plxna4
Fmod Pogin Pdpn $\quad$ Rel. $\begin{array}{ll}\text { Adam33 } & \text { Rel. } \\ \text { lgfbp5 } & \text { Expression }\end{array}$ Gm1284

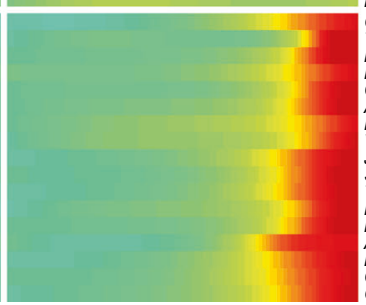

\section{d}
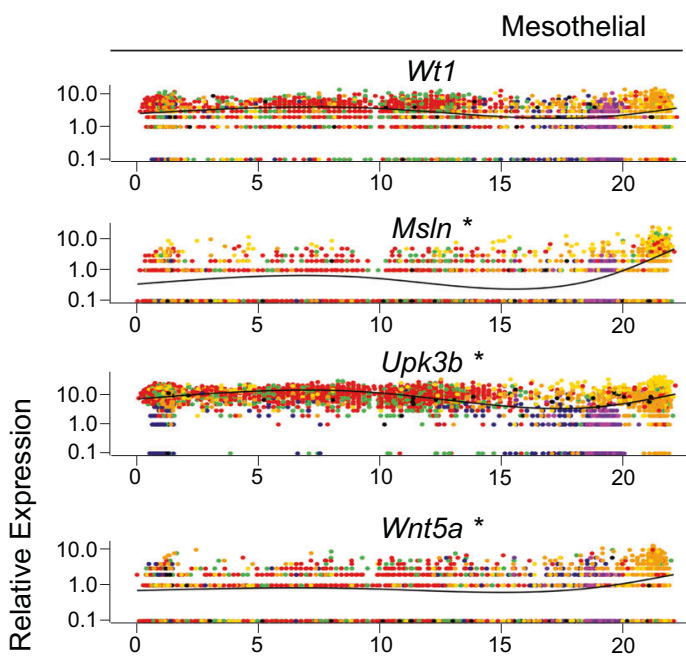

Sema3c *
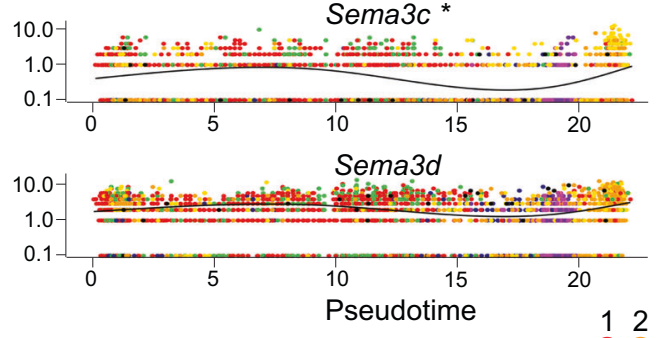

12345678
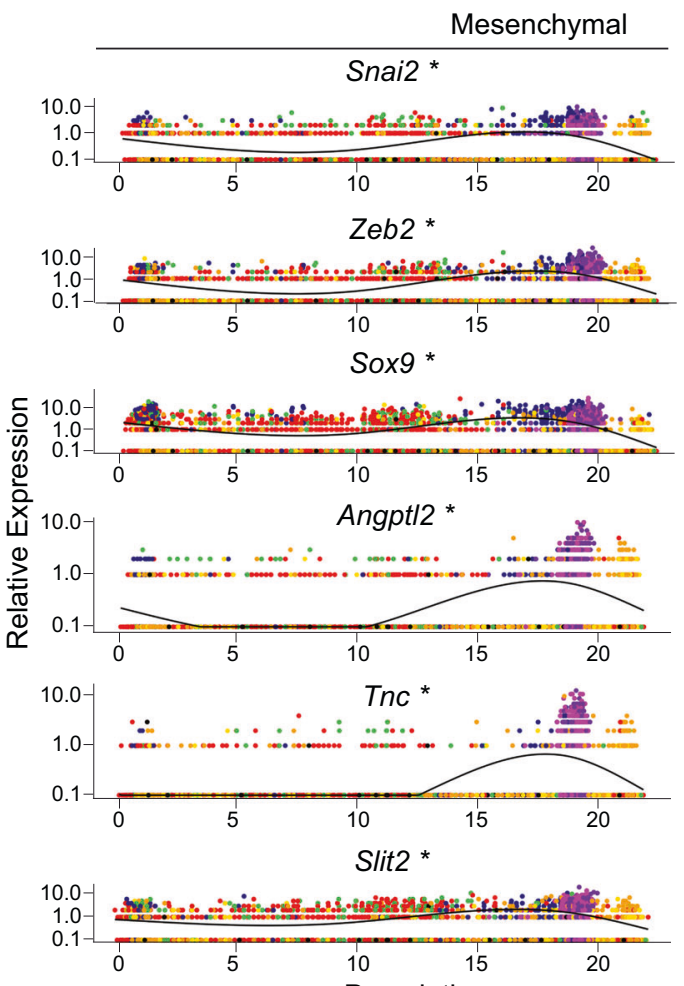

Pseudotime

Fig. 2 Epicardial-to-mesenchymal transition restricts expression of secreted ligands genes. a Monocle-generated pseudotime trajectory reveals the composition of three cell states based on the developmental stage (\%). Cells in state 1 are at the beginning of pseudotime. Cell states 2 and 3 diverge at a common branchpoint (BP). b Pseudotime trajectory reveals the composition of cell identity within cell states. State 1 contains epicardial progenitor cells from Cluster 1 (C1)/C3. State 2 is populated by mesothelial cells from C2/C4. State 3 emerges from a common progenitor through a transient state characterized by cells in C5 actively undergoing EMT and culminates in mesenchymal cells of C6/C7. c Hierarchical clustering visualization of genes that define pseudotime states. Relative (Rel.) expression represents the scaled expression of rlog normalized data. $\mathbf{d}$ Pseudotime-dependent genes augmented in mesenchymal versus mesothelial cells. Cells are colored according to cell cluster identity. ${ }^{\star}$ Genes with significant correlation with pseudotime state. 
enriched in EMT and mesenchyme genes such as Snai2, Zeb2, Sox9, Postn, and Ptn ${ }^{20}$ (Fig. 2c, d and Supplementry Fig. 6c). To further interrogate the angiogenic programs we identified in mature mesothelial and mesenchymal cells, we probed pseudotime kinetics for genes encoding axon guidance cues that regulate neuronal and vascular migration and pathfinding during development ${ }^{32}$. Among the more restricted guidance genes, we found epicardium-derived mesenchymal cells express an over-abundance of Angptl2, Tnc, and Slit2, whereas epicardium-derived mesothelial cells display an enrichment in Wnt5a, Sema3c, and Sema3d (Fig. 2d and Supplementary Fig. 6c; Supplementary Dataset 2).

To confirm that expression of Slit2 and Sema3d is enriched in epicardium-derived mesenchyme and mesothelium, respectively, we performed RNAscope multiplex fluorescence in situ hybridization (FISH) on heart sections obtained from Wt1 ${ }^{\text {CreERT2 }} ; \mathrm{R} 26 R^{\mathrm{mTmG}}$ embryos labeled at E9.5/E10.5 and collected at E12.5 (pre-EMT), E14.5 (mid-EMT), and E16.5 (post-EMT). Sema3d is observed broadly within $\mathrm{GFP}^{+}$ mesothelial cells on the epicardial surface at all time points examined (Fig. 3a, b and Supplementary Fig. 7a, b). In contrast, Slit2 is observed on the epicardial surface at E12.5, and is also expressed within a discrete population of epicardiumderived mesenchymal cells that are closely associated with Pecam1-expressing EC as early as E14.5, and most notably at E16.5 (Fig. 3a, c and Supplementary Fig. 7a, c). Of note, the expression of key angiogenic factors often increased in $\mathrm{GFP}^{+}$ EPDCs between E12.5 and E16.5, regardless of localization to mesothelial or mesenchymal cell populations (Supplementary Fig. $7 \mathrm{~d}$ ). The striking restriction of genes encoding secreted vascular guidance cues to distinct populations of EPDCs led us to speculate that angiogenic cues may be coordinately regulated with epicardial cell fate.

Disruption of epicardial EMT alters expression of vascular guidance cues. Myocardin-related transcription factors (MRTFs) are mechanosensitive transcriptional co-activators of serum response factor (SRF) that facilitate induction of cell contractility and motility gene programs ${ }^{33}$. We previously reported that epicardium-specific Cre-mediated deletion of Mrtf- $a$ and Mrtf- $b$ (MRTFepiDKO) or Srf (SRF ${ }^{e p i K O}$ ) during development impedes epicardial EMT, precipitating epicardium detachment and defects in coronary plexus formation and EC integrity ${ }^{7}$. This phenotype was attributed to a depletion of microvascular pericytes, which partially phenocopied the SRF-dependent emergence of mural cells from the epicardium ${ }^{19}$. In order to evaluate whether epicardial EMT controls the angiogenic programs identified by scRNA-seq, we used FACS to isolate GFP ${ }^{-}$non-EPDCs (enriched in myocytes), and $\mathrm{GFP}^{+}$EPDCs from wild-type (control) and mutant embryos for RNA-sequencing (Fig. 4a and Supplementary Fig. 8a-d). EPDCs obtained from MRTFepiDKO and SRFepiKO mice are transcriptionally indistinguishable based on principal component analysis (PCA), but diverge from control EPDCs stemming from the dysregulation of 2,518 genes (Fig. 4b, $c$ and Supplementary Fig. 9a, b). MRTFepiDKO EPDCs display a significant reduction in genes associated with biological processes such as cell migration (Mylk, Vin), ECM production (Colla2, Col3a1), and mesenchymal cell differentiation (Pdgfra, Acta2, Tagln) (Supplementary Fig. 9c-i). Surprisingly, MRTFepiDKO EPDCs also exhibit a significant downregulation of genes associated with paracrine regulation of chemotaxis and axon guidance (Supplementary Fig. 9c, j). Indeed, axon guidance was the most significantly dysregulated biological process in MRTFepiDKO EPDCs, represented by secreted ligands such as Efna5, Sema3d, Slit2, Slit3, and Wnt5a (Fig. 4c, d). qRT-PCR confirmed genes encoding select guidance cues are enriched in EPDCs, and significantly downregulated upon MRTF deletion
(Fig. 4e). RNAscope FISH also confirmed the reduction of Sema3d and Slit2 in EPDCs of MRTFepiDKO hearts, compared to controls (Supplementary Fig. 10a-c). These findings corroborated our scRNA-seq results revealing the restriction of vascular guidance cues to distinct populations of epicardium-derived cells, and provided evidence that EMT contributes to the expression and localization of these factors.

EMT regulates the expression of genes encoding vascular guidance cues. In order to further examine the effect of EMT on vascular guidance gene expression, we treated primary epicardial cells isolated from E11.5 embryos with TGF $\beta 1$ and PDGF-BB, which resulted in the downregulation of epicardial/mesothelial genes and upregulation of EMT-associated and mesenchymal genes (Fig. 5a) ${ }^{34}$. Sema3c and Sema3d were both significantly suppressed upon induction of epicardial EMT, whereas Tnc and Slit2 were upregulated (Fig. 5a). These gene expression changes are consistent with their in vivo distribution within mesothelial cells and mesenchymal cells, respectively. We also found evidence that EMT induces the mural cell phenotype based on the expression of pericyte marker genes Pdgfrb and Cspg4 (Fig. 5a). We, therefore, re-evaluated EPDC populations 5, 6, and 7 (from Fig. 1) to establish the identity of Wt1-lineage mesenchymal cells and define the source of epicardium-derived guidance cues (Fig. 5b). We were able to identify fibroblasts (Fb-1, Fb-2, Acta2 ${ }^{+}$ $\mathrm{Fb}$ ) based on increased expression of Col1a1, Postn, and Tnc; smooth muscle cells (SMC-1, SMC-2) based on increased expression Tagln; and pericytes (PC) based on increased expression of Pdgfrb (Fig. 5c). Slit2 and Angptl2 are enriched in FB1 and FB2, and Slit2 is especially pronounced in pericytes (Fig. 5c). The Cspg4 ${ }^{\mathrm{CreERT2}}$ mouse line has been used to lineage trace vascular mural cells, including pericytes ${ }^{35}$. FISH using probes against Gfp and Slit2 on heart sections obtained from Cspg4 ${ }^{\mathrm{CreERT}} ; \mathrm{R} 26 \mathrm{R}^{\mathrm{mTmG}}$ embryos obtained at E17.5 revealed Slit2 transcripts within some Cspg4 lineage-derived mural cells (Fig. 5d). Collectively, these data describe a paradigm whereby epicardial EMT is responsible for the restriction of individual chemotactic cues to distinct epicardium-derived lineages, including coronary mural cells, which may represent a vascular guidance cell reminiscent of the guidepost neuron ${ }^{16}$.

Single-cell transcriptomics defines the EC response to epicardial dysfunction. Coronary EC re-specification into arterial and venous fates occurs at around E14.5 ${ }^{1,2}$. In order to interrogate the impact of epicardial EMT on individual ECs, we isolated $\mathrm{CD} 31^{+} / \mathrm{CD} 45^{-}$cells from MRTFepiDKO and Control hearts at E14.5 by FACS followed by single-cell capture and scRNA-sec using the $10 \times$ Genomics platform (Fig. $6 a$ and Supplementary Figs. $11 \mathrm{a}-\mathrm{c}$ and $12 \mathrm{a}, \mathrm{b})$. CCA defined 9 unique EC populations that were enriched in Pecam1 (Supplementary Fig. 13a, b), and alleviated concerns of batch effects based on genotype and cell cycle analysis (Supplementary Fig. 13c, d; Supplementary Dataset 3 and 4). Since cell cycle is reported to underlie transcriptional differences during EC differentiation ${ }^{9,36}$, we performed unbiased clustering without regression of cell cycle to allow for identification of EC phenotypes that emerge upon disruption of epicardial EMT (Fig. 6b and Supplementary Fig. 13e). This analysis defined 9 unique cell populations consisting of ECs categorized as sinus venosus (SV), coronary plexus, angiogenic, venous, arterial, endocardial, and general endothelial (Fig. 6b). UMAP plots of filtered and typed ECs showed that cell clusters C3-C5 and C9 were significantly enriched with MRTFepiDKO ECs (Fig. 6b, c and Supplementary Fig. 13f, g). Violin gene expression plots defined MRTFepiDKO-enriched sub-populations of SV and coronary plexus ECs that exhibit differential expression of Aplnr, Apln, and 
a
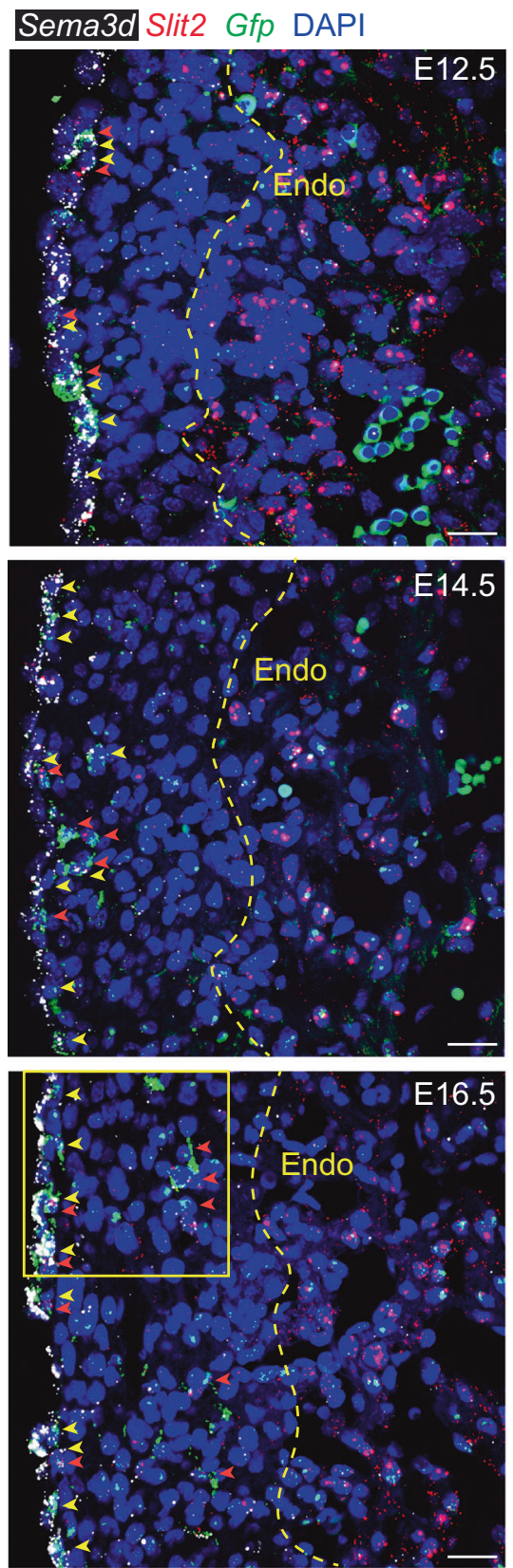

a'

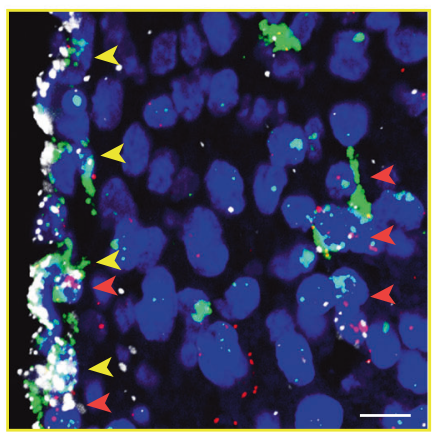

b

Pecam1 Sema3d Gfp DAPI
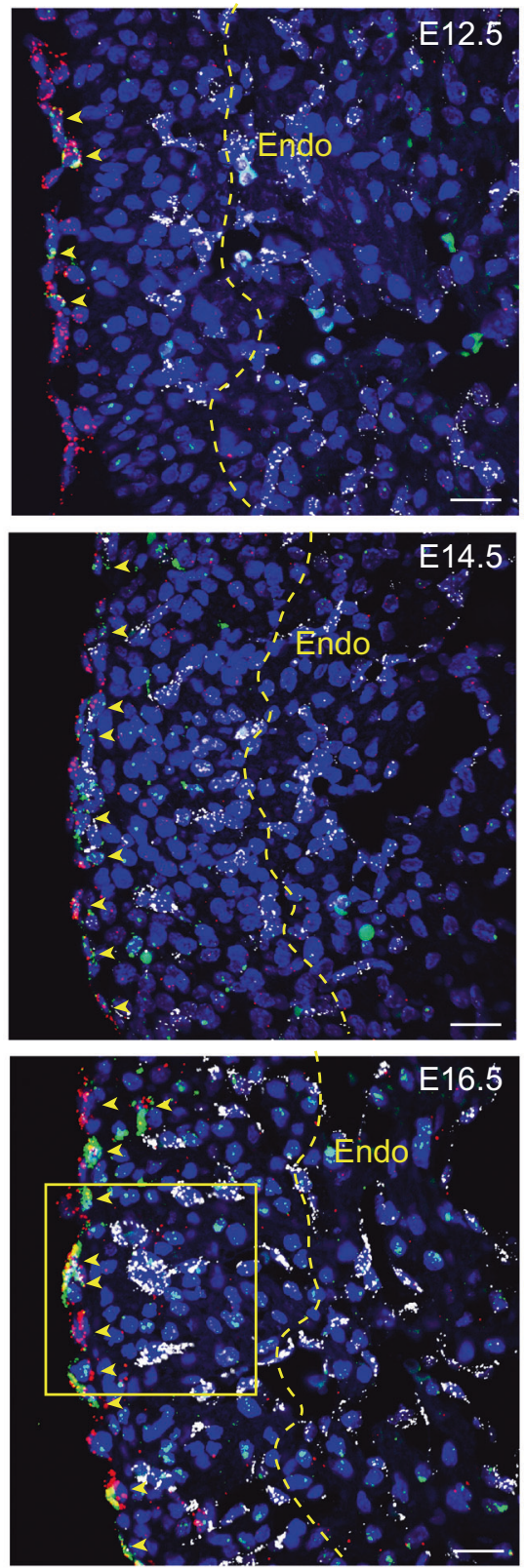

b'

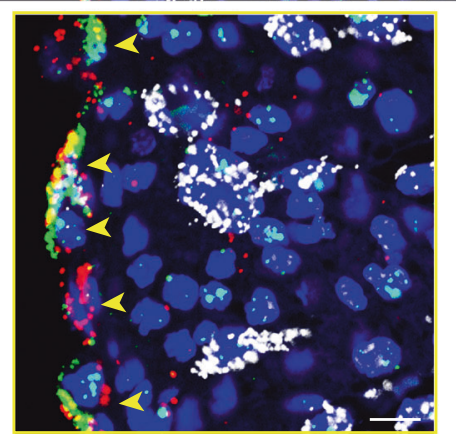

c
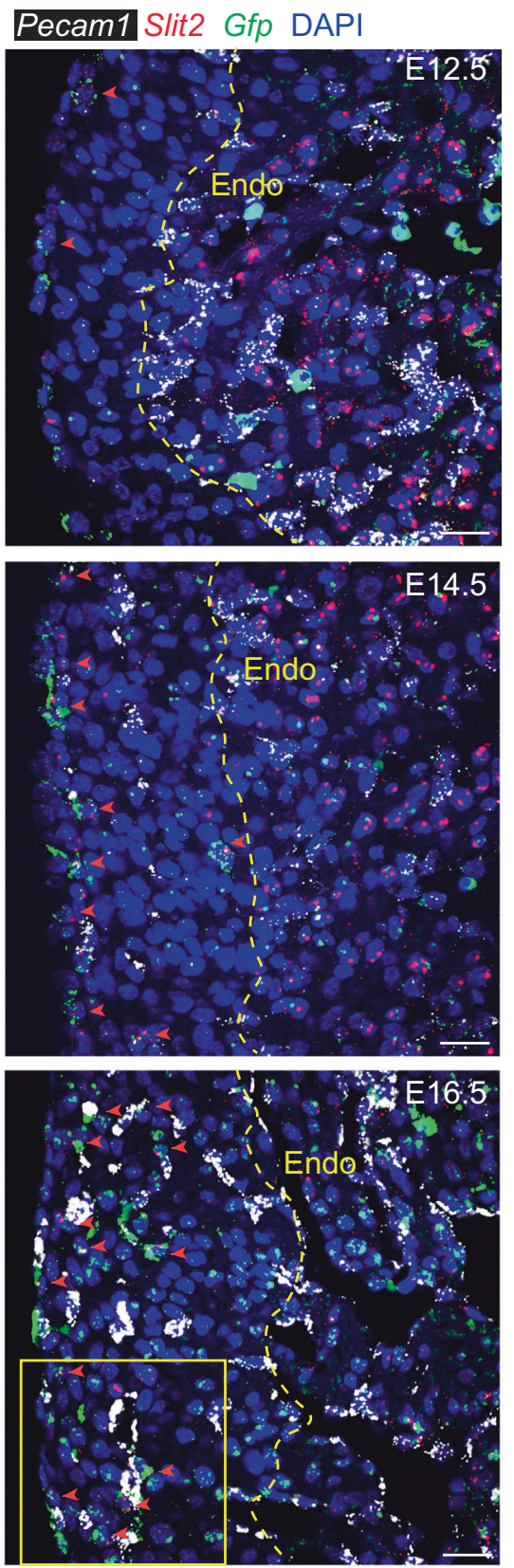

c'

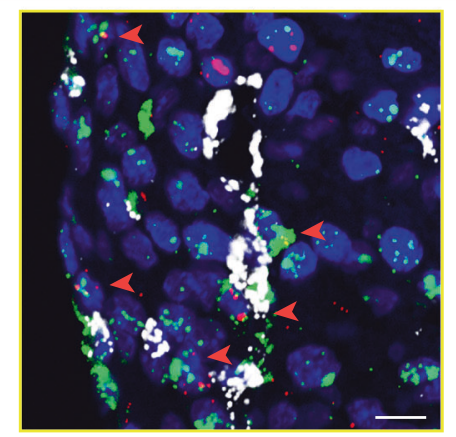

Fig. 3 In situ analysis confirms the expression and localization of vascular guidance genes in epicardium-derived cells. a FISH using probes against Gfp (green) to detect epicardium-derived cells and Sema3d (white) or Slit2 (red) reveals diverging localization of vascular guidance cues within the epicardium and interstitium between embryonic (E)12.5 and E16.5. $\mathbf{a}^{\prime}, 3 \times$ zoom of E16.5. b Expression of Sema3d (red) in Wt1 lineage-derived cells (Gfp ${ }^{+}$, green) relative to

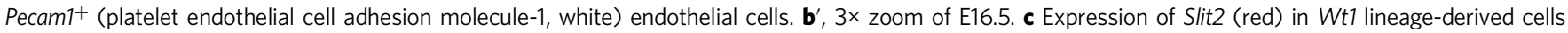
$\left(\mathrm{Gfp}^{+}\right.$, green) relative to Pecam7 ${ }^{+}$(white) endothelial cells. $\mathbf{c}^{\prime} 3 \times$ zoom of E16.5. Yellow arrowhead, Gfp ${ }^{+} / \mathrm{Sema} \mathrm{d}^{+}$cells. Orange arrowhead, Gfp ${ }^{+} / \mathrm{Slit2}^{+}$cells. Dashed yellow line represents the endocardium-myocardium border. DAPI staining was utilized to visualize nuclei (blue). Scale bar, $20 \mu \mathrm{m}$. Scale bar $3 \times$ zoom, $10 \mu \mathrm{m}$. Endo endocardium. 


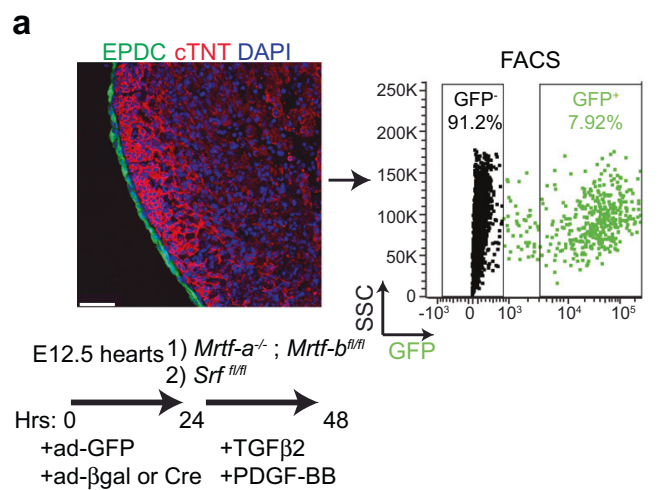

c

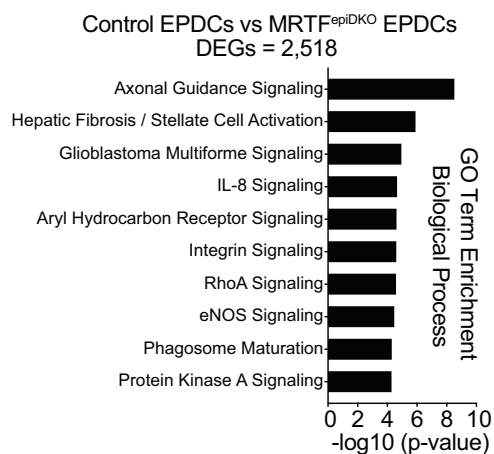

d

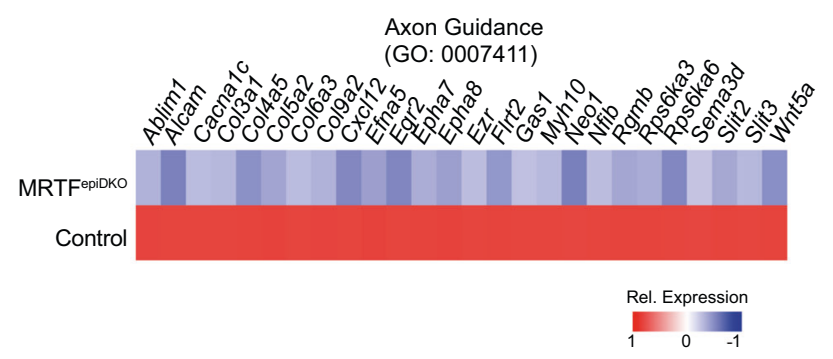

e

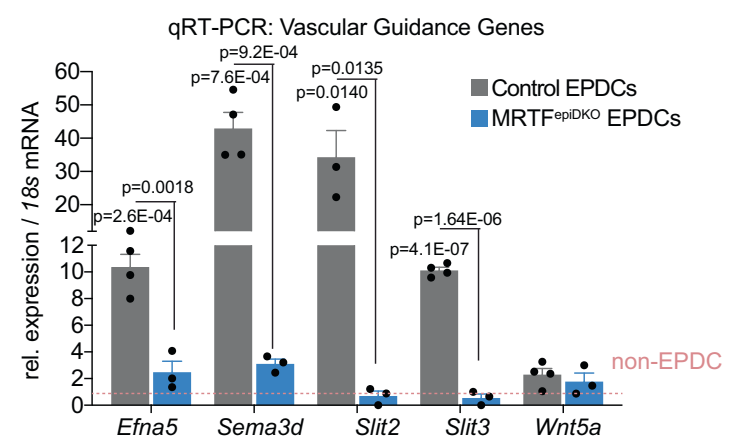

Fig. 4 Genetic disruption of epithelial-to-mesenchymal transition alters the expression of vascular guidance cues. a Schematic of experimental design to isolate epicardial cells (EPDC) of indicated genotypes for bulk RNA-seq. Embryonic day (E)12.5 hearts were removed from Mrtf-a-/-;Mrtf-bfl/fl,

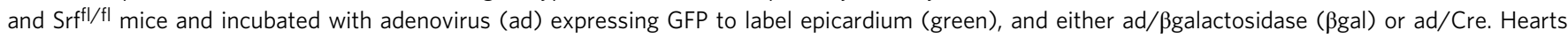
were cultured ex vivo in TGF $\beta 2[2 \mathrm{ng} / \mathrm{mL}]$ and PDGF-BB $[20 \mathrm{ng} / \mathrm{mL}]$ to induce EMT, and GFP+ epicardial cells were collected using FACS. GFP- cells were collected as non-EPDCs. cTNT, cardiac troponin T (red), and DAPI staining were utilized to visualize nuclei (blue). Scale bar, $50 \mu \mathrm{m}$. Refer to Supplementary Fig. 8e for FACS sequential gating and enrichment of epicardial cells. b Principal component analysis (PCA) of GFP- non-EPDCs and GFP+ EPDCs from control, Mrtf-a;Mrtf-b double knockout (MRTFepiDKO) or Srf knockout (SRFepiKO) mice. c Analysis of differentially expressed genes (DEGs) reveals biological processes that are enriched between MRTFepiDKO and control EPDCs. GO-term enrichment significance was determined using Fisher exact test. d Heat map highlighting the relative expression of genes involved in axon guidance signaling in EPDCs of the indicated genotype. Relative (Rel.) expression represents the scaled expression of rlog normalized data. e Expression of genes encoding axon guidance cues was validated by qRT-PCR. Values are represented as a fold change in expression relative to non-EPDCs (dashed line at 1). $n$ represents samples acquired from independent embryos, which were analyzed in 1 experiment. $n=3$ non-EPDCs, $n=4$ for Efna5, Sema3d, Slit3, Wnt5a, and $n=3$ for Slit2 in Control EPDCs; $n=3$ MRTFepiDKO EPDCs were analyzed. Data are presented as mean values \pm SEM. Statistical significance was determined by a two-sample unpaired Student's $t$-test. TGF $\beta 2$ transforming growth factor beta-2, PDGF-BB platelet-derived growth factor BB, m-tdTom membrane tdTomato, m-eGFP membrane-enhanced green fluorescent protein, TAM tamoxifen.

the proliferative marker $C d k 1^{9,37}$ (Fig. 6d). Angiogenic genes Sparcll and $C d 47^{38}$ and pre-arterial genes Dll4 and Sox $17^{9}$ were also enriched in MRTFepiDKO dominated clusters (Supplementary Fig. 14a-c). However, genes associated with the endocardium $(N p r 3, N f a t c 1)^{8,39}$, venous $(N r 2 f 2)^{9}$ or mature arterial (Gja4, Gja5, Fbln 5$)^{9,38}$ were similarly expressed in UMAP clusters of Control and MRTFepiDKO ECs (Fig. 6d and Supplementary Fig. 14c, d). Overall, these data reveal that disruption of epicardial EMT leads to the emergence of a population of ECs that exhibit an immature vascular cell phenotype.

Identification of potential receptor-ligand pairings between the endothelium and epicardium. Coordination of cell-to-cell signaling is critical to building vascular networks and underlies the acquisition of arterial and venous cell identity. To characterize the intercellular signaling between EPDCs and coronary ECs that influence developmental angiogenesis, we constructed a receptor-ligand visualization by matching EC-expressed receptors to epicardium-derived ligands identified by scRNA-seq, indicating whether a particular ligand is enriched within epicardium-derived mesothelial or mesenchymal cells (Supplementary Fig. 15a). We then identified epicardium-derived vascular guidance genes that are influenced by epicardial EMT by cross-referencing to our bulk RNA-sequencing of MRTFepiDKO EPDCs (Supplementary Fig. 16a and Supplementary Dataset 5). We identified 99 genes encoding ligands that are dysregulated upon Mrtf deletion, potentially impacting pathways related to proliferation, growth factor signaling, non-canonical Wnt signaling, ECM-receptor interactions, and axon guidance (Supplementary Fig. 16a-c); a total of 52 ligands that are normally restricted to the mesothelium or mesenchyme in control mice are disrupted (Supplementary Fig. 17a-c). Notably, 34 genes encoding receptors that are detected in ECs were significantly altered following Mrtf deletion within the epicardium (Supplementary Fig. 18), which led to the potential disruption of 87 receptor-ligand pairs (Supplementary Fig. 15b and Supplementary Dataset 6).

We next re-evaluated the single EC transcriptome and conducted RNA FISH to evaluate the distribution of key cell surface receptors within the fetal heart, focusing on candidate receptors for Sema3d (mesothelium-derived) and Slit2 (guidepost cell-derived). We detected the semaphorin co-receptor $N r p 1$ within all EC clusters (Fig. 6e). RNA FISH confirmed the widespread expression 
a

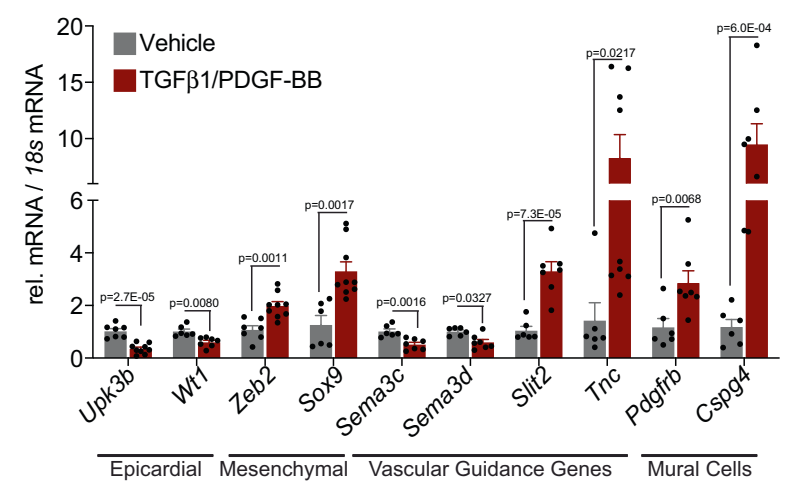

C
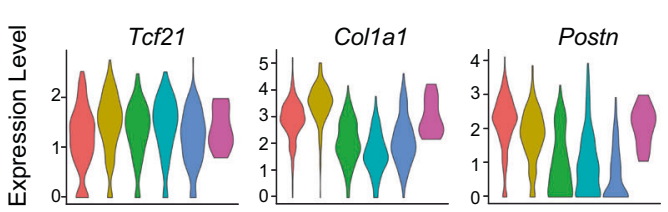

FB-1

SMC- -2

SMC-2

- Acta2 ${ }^{+} \mathrm{FB}$
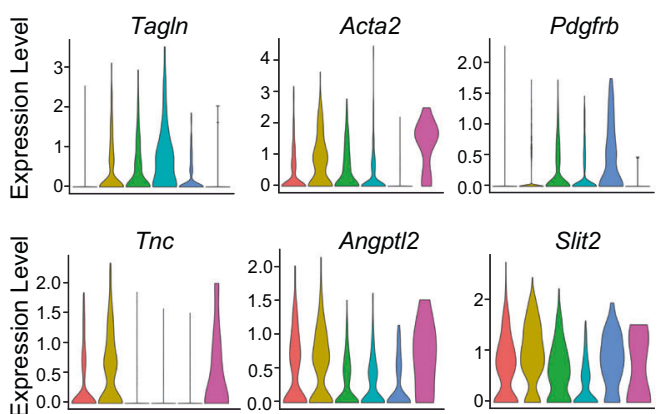

b

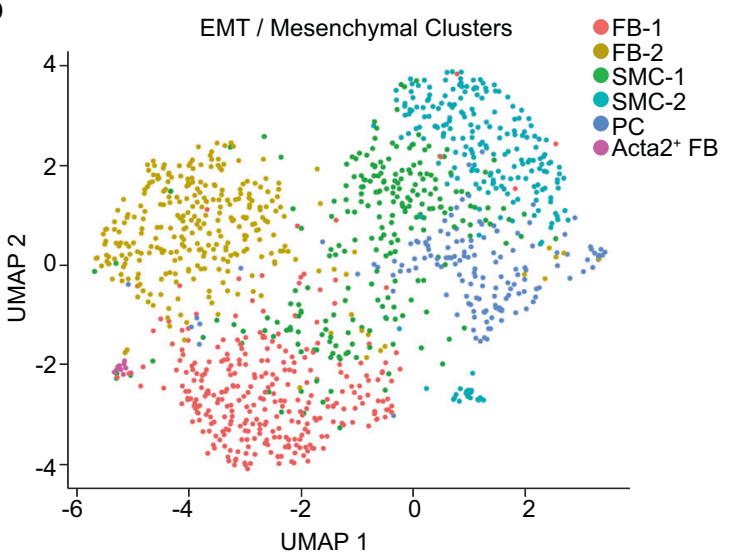

d
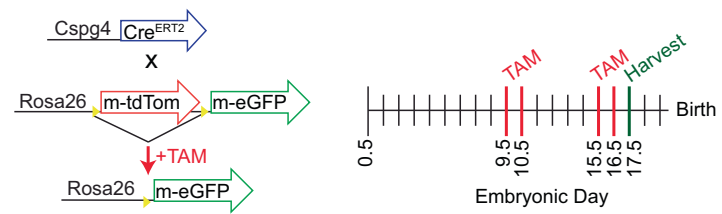

Pecam1 Slit2 Gfp DAPI
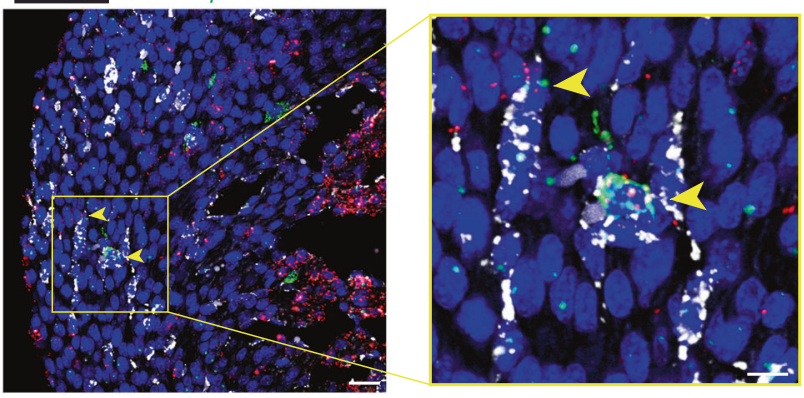

Fig. 5 Vascular guidance cues are differentially expressed upon in vitro induction of EMT. a Primary epicardial cells were cultured \pm TGF $\beta 1$ [10 ng/mL] and PDGF-BB $[20 \mathrm{ng} / \mathrm{mL}]$ to induce EMT. Relative expression of genes encoding axon guidance cues was evaluated by qRT-PCR, along with markers of epicardial, mesenchymal, and mural cells. $n$ represents samples isolated from independent embryos, which were analyzed over 2 experiments. Vehicle $n=$ 6 for Sox9, Sema3c, Tnc and $n=7$ for Upk3b, Wt1, Zeb2, Sema3d, Slit2, Pdgfrb, Cspg4; and TGFp1/PDGF-BB $n=7$ for Wt1, Sema3c, Sema3d, Slit2, Pdgfrb, Cspg4 and $n=9$ for Upk3b, Zeb2, Sox9, Tnc. Data are presented as mean values \pm SEM. Statistical significance was determined by a two-sample unpaired Student's t-test. b Seurat was used to re-cluster cells undergoing EMT (cluster 5) and mesenchymal cells (clusters 6/7) defined in Fig. 1f. c Violin plots representing select genes associated with fibroblast or mural cell identity. Expression level represents log normalized expression. $\mathbf{d}$ Experimental strategy for lineage tracing vascular mural cells in Cspg 4 CreERT2;R26 $\mathrm{mTmG}$ embryos. FISH was performed on hearts with probes directed against Gfp (green) and Slit2 (red) as demonstrated in low (left) and high (right) magnification images and relative to Pecam1+ (platelet endothelial cell adhesion molecule-1, white) endothelial cells. Yellow arrowheads, Slit2 expression in Cspg4 lineage-derived cells. Scale bar, $25 \mu \mathrm{m}$ (left image) and $10 \mu \mathrm{m}$ (zoom right image). DAPI staining was utilized to visualize nuclei (blue). Immunostaining was repeated independently 3 times with similar results. EMT epithelial-to-mesenchymal transition, TAM tamoxifen, FB fibroblast, SMC smooth muscle cell, PC pericyte.

of Nrp1 within a majority of Pecam1-expressing ECs, while Sema3d is restricted to mesothelial cells on the cardiac surface (Fig. $6 \mathrm{f}$ and Supplementary Figs. 19, 20a). In contrast, Nrp2 and Robo4 are primarily restricted to angiogenic and coronary plexus EC within clusters 2, 5, and 9 (Fig. 6e). FISH confirmed the expression of Robo4 within a distinct population Pecam1 ${ }^{+}$ECs that often reside in close proximity to Slit2 ${ }^{+}$cells (Fig. $6 \mathrm{~g}$ and Supplementary Figs. 19, 20b). These data reveal the potential for complex intercellular cross-talk between EPDC and EC that may influence coronary angiogenesis.

Disruption of epicardial EMT alters EC developmental trajectory. To define how EC developmental trajectory is altered upon disruption of epicardium-derived paracrine signaling, we ordered ECs obtained from control and MRTFepiDKO mice at E14.5 in pseudotime using Monocle (Supplementary Fig. 21a and
Supplementary Dataset 7). While each pseudotime state is composed of ECs obtained from animals of both genotypes, states 5 and 7 are enriched in ECs from control mice, and states 1-4 are enriched in MRTFepiDKO ECs (Fig. 7a and Supplementary Fig. 21b, c). Cell state also correlates with cell cycle activity (Fig. 7b), consistent with reports that EC maturation coincides with reduced proliferation ${ }^{9,36}$. Pseudotime originates in state 1 with SV and angiogenic coronary progenitors, marked by the expression of Aplnr, Apln, and Sparcl1, and an over-abundance of cells in the G2/M and S phases of the cell cycle (Fig. 7b, c). ECs obtained from control hearts primarily followed a trajectory from progenitor state 1 through a mixed venous/primed arterial EC state 5 (represented by $N r 2 f 2, E p h b 4, D a b 2$ ) towards terminal states 6 and 7. State 6 displays immature arterial-like characteristics, with relatively lower levels of the venous markers $N r 2 f 2$ and Ephb4, elevated levels of the early arterial gene Efnb2, and 
a

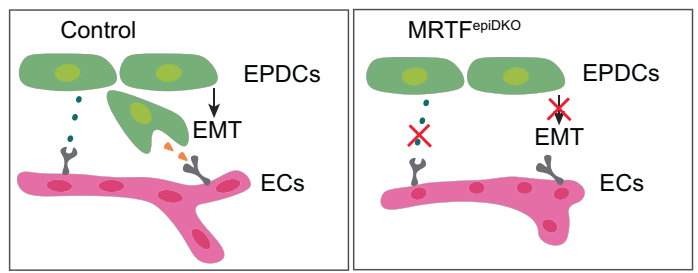

b

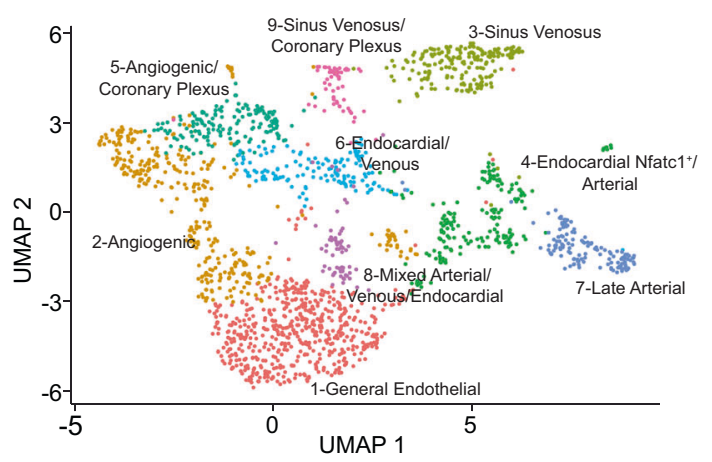

C

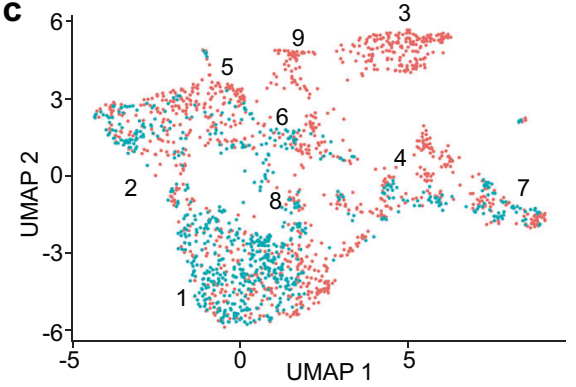

d

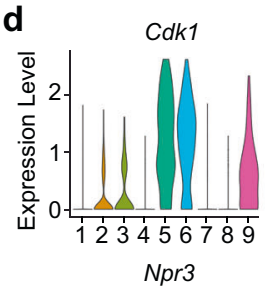

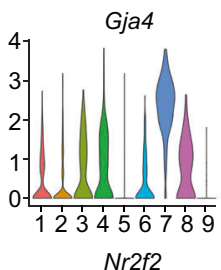

$N r 2 f 2$

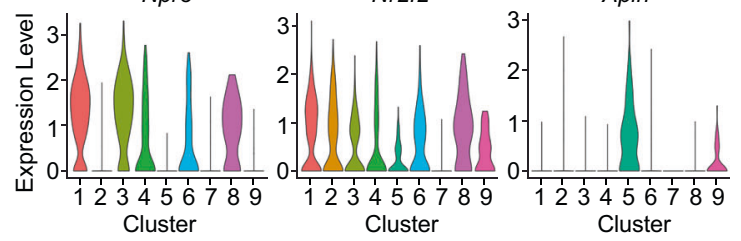

EC - Control

EC - MRTF

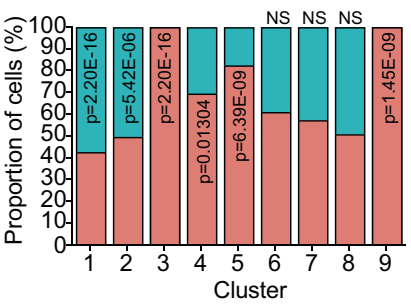

e
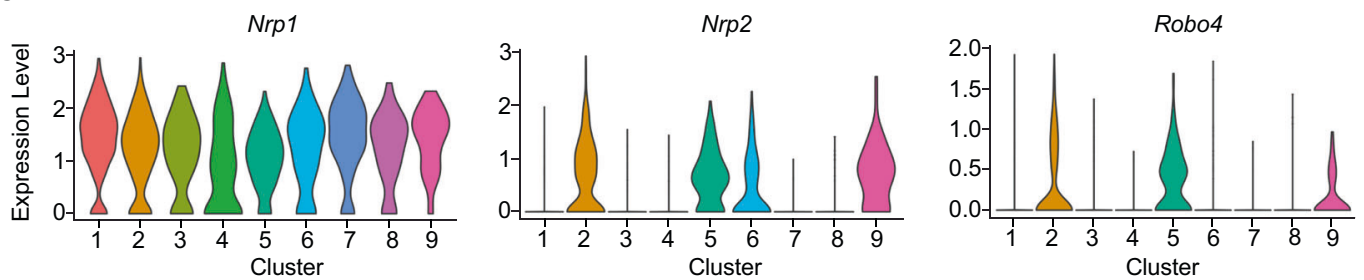

f
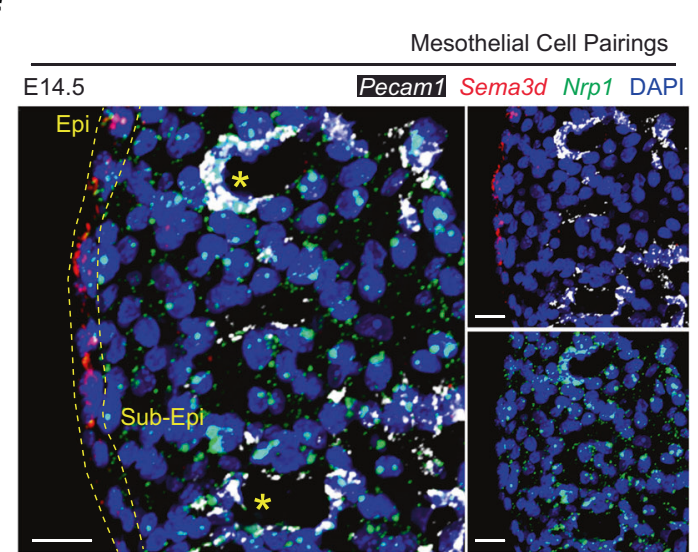

g
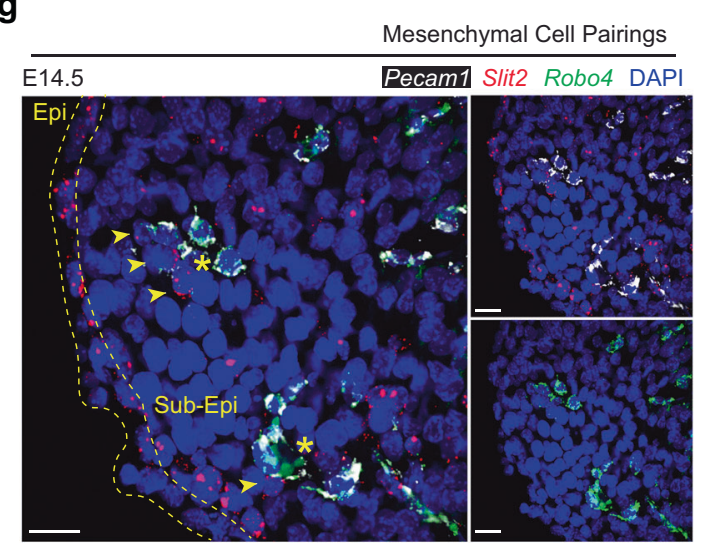

Fig. 6 Disruption of epicardial EMT alters intercellular cross-talk between the epicardium and coronary vasculature. a Schematic of vasculature development representing disruption of intercellular signaling hypothesized in Mrtf-a;Mrtf-b double knockout (MRTFepiDKO) hearts. b, c UMAP representation of single-cell transcriptomes of embryonic day (E) 14.5 endothelial cells (ECs) represented by $\mathbf{b}$ cell identities and $\mathbf{c}$ genotype with the proportion of each cell cluster (\%) contributing to either the Control or MRTFepiDKO genotype is graphed on the right. A two-sample Student's $t$-test was performed to determine the significance of the proportion of cells in single-cell clusters. Refer to Supplementary Fig. 11b, c for FACS sequential gating and enrichment of ECs. d Violin plots showing expression of select genes associated with EC identity. e Violin plots showing expression of select receptors for Sema3d and Slit2 ligands in the 9 EC clusters. Expression level in $\mathbf{d}$ and $\mathbf{e}$ represents log normalized expression. $\mathbf{f}, \mathbf{g}$ Expression of select ligand-receptor pairs within epicardial cells and Pecam1+ ECs (platelet endothelial cell adhesion molecule-1, white) at E14.5 visualized by FISH.

f Mesothelial ligand Sema3d (red) is observed on the epicardial surface and Nrp1 (green) expression is in ECs. $\mathbf{g}$ The mesenchymal enriched ligand Slit2 (red) is expressed in interstitial epicardial cells, often associated with Robo4-positive (green) ECs. Yellow asterisks, developing coronary vessels. Yellow arrowheads, Slit2 expressing cells. Dashed lines signify the epicardial border. Scale bars, $20 \mu \mathrm{m}$. DAPI staining was utilized to visualize nuclei (blue). Immunostaining was repeated independently 3 times with similar results. NS not-significant, EMT epithelial-to-mesenchymal transition, Epi epicardium, Sub-Epi sub-epicardium. 
a

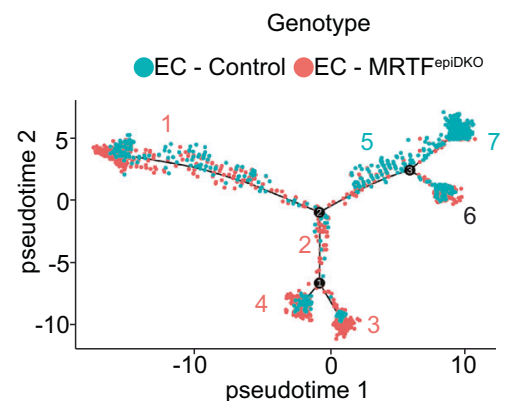

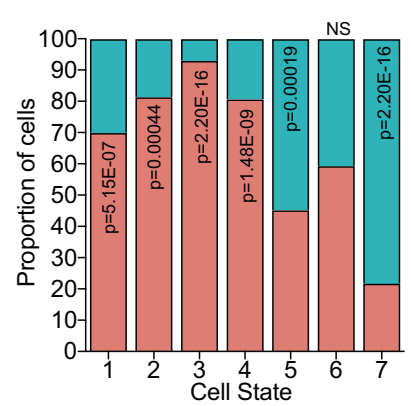

b Cell Cycle Activity

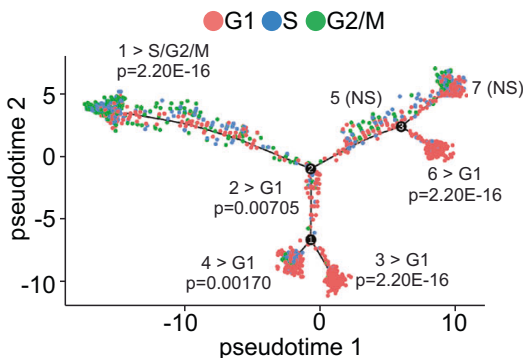

C
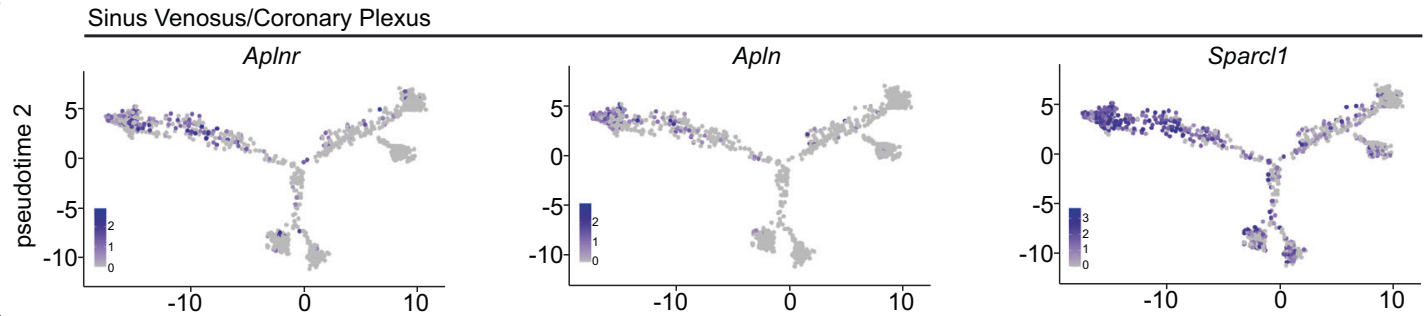

d

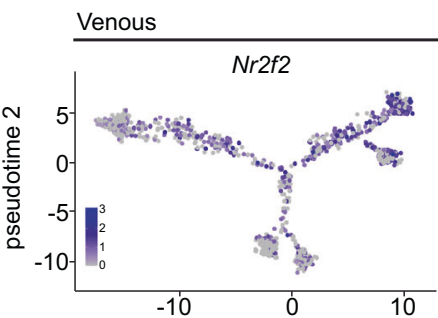

e
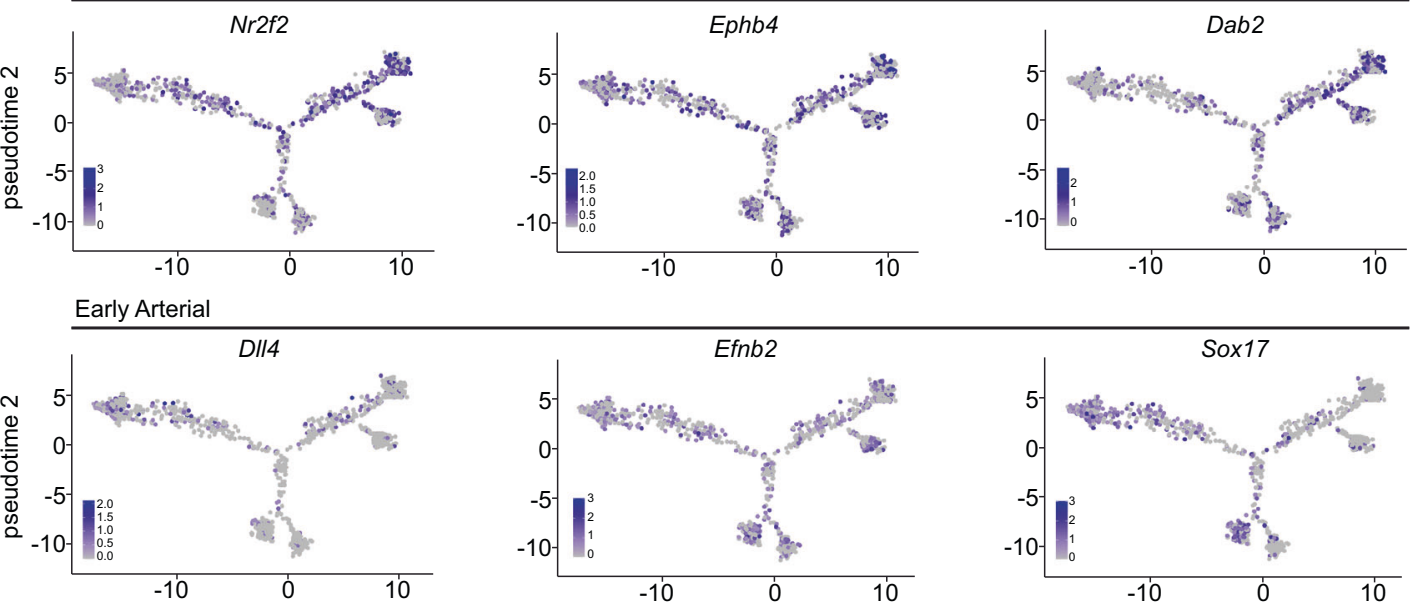

f
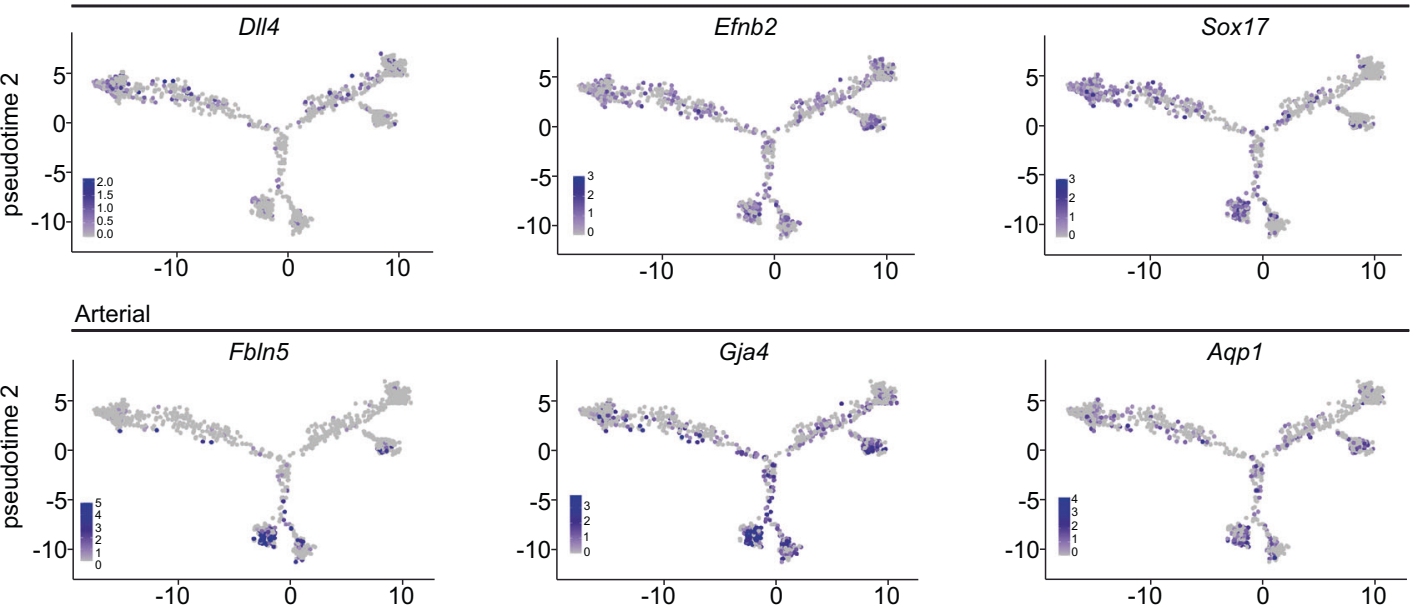

g
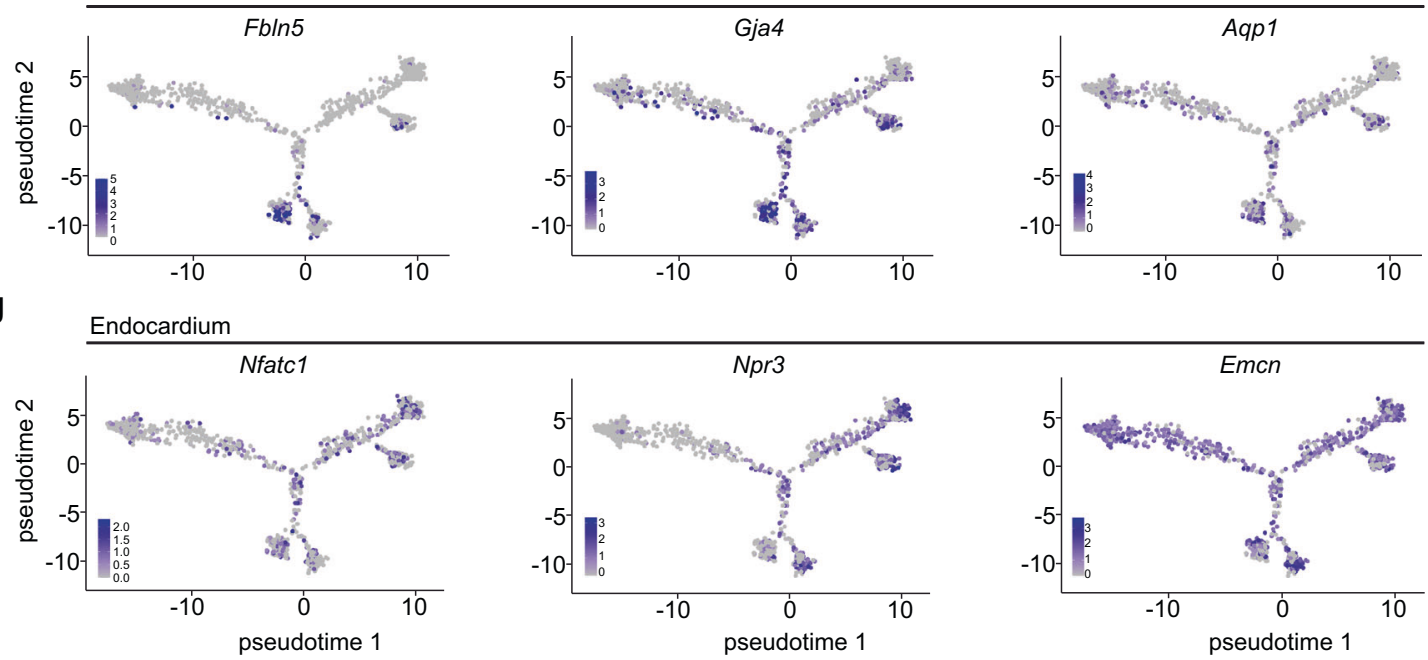

Fig. 7 Disruption of epicardial EMT alters arteriovenous differentiation and maturation. $\mathbf{a}$, $\mathbf{b}$ Monocle-generated pseudotime trajectory of ECs from control and Mrtf-a;Mrtf-b double knockout (MRTFepiDKO) hearts. Developmental trajectories represented by a genotype with the proportion (\%) of control or MRTFepiDKO ECs represented in each cell state (right) and $\mathbf{b}$ cell cycle activity with a representation of cells in the G1, S, or G2/M phase of the cell cycle. A twosample Student's t-test was performed to determine significance of the proportion of cells in single-cell clusters presented in $\mathbf{a}$ and $\mathbf{b}$. c-g Pseudotime feature plots representing the expression of genes related to the $\mathbf{c}$ sinus venosus/coronary plexus, $\mathbf{d}$ venous, $\mathbf{e}$ early arterial, $\mathbf{f}$ late arterial, and $\mathbf{g}$ endocardial cell identity. The scale represents relative (Rel.) expression. NS not-significant, EMT epithelial-to-mesenchymal transition. 
intermediate levels of the mature arterial gene marker Gja4 (Fig. $7 \mathrm{~d}-\mathrm{f}$ and Supplementary Fig. 22a, b). In contrast, terminal state 7 is represented by cells with more venous-like characteristics, expressing higher levels of $\mathrm{Nr} 2 \mathrm{f2}$, Ephb4, and Dab2 (Fig. 7d and Supplementary Fig. 22a, b). The start of pseudotime is enriched in ECs obtained from MRTFepiDKO hearts, which transition through a unique developmental trajectory (states 2-4), characterized by ECs that have exited the cell cycle (Fig. 7a, b). State 2 represents a transient EC phenotype leading towards state 4, which is defined by early (Efnb2, Sox17) and mature (Fbln5, Gja4, and Aqp1) arterial markers (Fig. 7e, f and Supplementary Fig. 22a, b). Cell state 3 is composed nearly entirely of ECs from MRTFepiDKO hearts that have exited the cell cycle and express intermediate levels of angiogenic and arterial genes as well and endocardial markers (Fig. 7f, g and Supplementary Fig. 22a, b). Endocardial gene markers Nfatcl and Npr3 are most enriched in terminal states 3, 6, and 7, whereas Emcn was detected broadly across cells ordered in pseudotime (Fig. $7 \mathrm{~g}$ and Supplementary Fig. 22a, b). Collectively, ECs from MRTFepiDKO hearts lack obvious venous identity markers and show a bias towards immature arterial fate, indicating epicardial EMT-dependent EPDC-EC signaling may directly impact EC maturation.

Disruption of epicardial EMT alters EC maturation and localization. Coronary vessel maturation requires the re-specification of immature ECs towards arterial fate in deeper myocardium, and venous fate in sub-epicardial myocardium. To begin investigating the potential impact of guidepost cell-derived ligands on coronary EC differentiation, we used an adenoviral-vector to express Slit2 in E13.5 hearts cultured ex vivo (Fig. 8a and Supplementary Fig. 23a). This method allowed for specific targeting of Slit2, or GFP control, to the epicardium (Fig. 8b). Following $24 \mathrm{~h}$ of infection, $\mathrm{CD} 1^{+} \mathrm{ECs}$ were isolated from hearts using FACS (Fig. 8a), and EC differentiation and maturation markers were evaluated by qRT-PCR. Slit 2 overexpression led to a reduction in the expression of arterial markers Gja4, Efnb2, and Apln, and increased expression of the venous/angiogenic endothelium marker Aplnr, although key venous markers (Nr2f2 and Ephb4) were unchanged ${ }^{9,38,40,41}$ (Fig. 8c-f and Supplementary 23b, c). Our data are consistent with reports of Slit2 regulating angiogenesis ${ }^{42,43}$, and suggest a common mechanism may guide vascular pathfinding and arteriovenous fate specification.

EC maturation is also characterized by cell polarization, induced by the connection of the nascent vasculature to arterial blood flow, which increases hemodynamic shear stress and supports EC alignment and migration against flow ${ }^{44}$. In order to evaluate EC maturation and polarization, we immuno-stained sections from control and MRTFepiDKO hearts obtained at E14.5 and E17.5 with antibodies directed against EMCN and ERG, a pan-EC ETS-family transcription factor ${ }^{45}$. The length-to-width ratio of $\mathrm{ERG}^{+}$nuclei was quantified as an indicator of cell polarity, revealing considerable elongation between E14.5 and E17.5 in control hearts, an alteration that was not observed in $\mathrm{ERG}^{+}$nuclei of MRTFepiDKO hearts (Fig. 9a-c). Quantification of $\mathrm{ERG}^{+}$nucleus localization also revealed an inappropriate accumulation of ECs near the epicardial surface in MRTFepiDKO hearts at E14.5 and E17.5 (Fig. 9a, d, e and Supplementary Fig. 24a, b). To further interrogate the distribution of mature ECs, immunostaining was performed to visualize venous EC (EMCN, green) and arterial EC (Cx40, red), which display sub-epicardial and mid-myocardial localization in control E17.5 hearts. In contrast, $\mathrm{EMCN}^{+}$venous cells displayed an abnormal accumulation near the epicardial surface in MRTFepiDKO hearts (Fig. 9f, g; Supplementary Fig. 25). We also often found $\mathrm{Cx} 40^{+}$arterial EC as discontinuous patches of cells without a discernable lumen, which resided deeper within the myocardium of MRTFepiDKO hearts (Fig. 9f, h; Supplementary Fig. 25). Collectively, these findings reveal a contribution of epicardium-derived pathfinding cues to EC localization and AV specification.

\section{Discussion}

In summary, our data establish epicardial EMT as a driving force in the generation of distinct expression domains of vascular patterning cues characterized by: (1) Mesothelial cells on the surface of the heart expressing angiogenic chemokines such as Sema3d; and (2) Epicardium-derived mesenchymal cells that express chemokines such as Slit2 and Angptl2. Our data also reveal the coordinated regulation of coronary $\mathrm{EC}$ localization and $\mathrm{AV}$ specification by epicardium-derived vascular patterning cues.

We previously reported that deletion of MRTFs in the epicardium prevents EMT, and inhibits coronary plexus formation ${ }^{7}$. The current transcriptome analyses further establish the epicardium as an important source of vascular guidance cues in the embryo, which is disrupted in MRTF mutant mice. Here, we define the specific role of epicardial EMT in establishing the spatial pattern of vascular cues that control EC patterning. We found that EMT induces the expression of secreted ligands that are found in epicardium-derived mesenchyme, while silencing those ligands that are restricted to the mesothelium. Slit2 is especially induced upon epicardial EMT, and localizes to a minor population of epicardium-derived fibroblasts and pericytes that we term vascular "guidepost cells". This population is reminiscent of the guidepost neuron in axon patterning, which provides noncontinuous landmarks that act as "stepping stones" for growing axons ${ }^{16}$. While the regulation of vascular guidance molecules seems largely dependent on EMT, reduction of the mesotheliumrestricted Sema3d in MRTF mutant mice suggests general epicardial dysfunction, supported by the suppression of canonical epicardial genes Aldh1a2, Tbx18, Tcf21, and $W t 1^{15,46,47}$.

Prior studies have revealed the importance of individual factors such as Sema3d and Slit2 in patterning of coronary venous cells and supporting cardiomyocyte cytokinesis ${ }^{48,49}$. Here, we found Slit $2^{+}$guidepost cells in close proximity to Robo4 ${ }^{+}$ECs in the sub-epicardium; thus, Slit2-Robo4 interactions are positioned to control angiogenesis and vascular stability, as described in other contexts $37,43,50,51$. Indeed, our study found that overexpression of Slit2 suppressed the arterial EC phenotype in ex vivo heart culture, based on the expression of arterial (Gja4 and Efnb2) and angiogenic venous markers (Aplnr). This result is consistent with the accumulation of ECs that exhibit an immature arterial phenotype upon suppression of Slit2 expression in MRTFepiDKO hearts. However, $\mathrm{Cx} 40^{+}$arterial ECs become mislocalized and fail to consistently form lumens in MRTFepiDKO embryos at E17.5, revealing a defect in EC maturation. Evidence for improper arterial cell differentiation upon epicardial disruption is consistent with the retention of a sinus venosus and coronary plexus EC phenotype, represented by the expression of Aplnr, Apln, $V e g f a, V e g f c, C d 47$. Of note, AV specification is in part regulated by COUP-TFII (also known as Nr2f2), which inhibits Notch activity in ECs and blocks differentiation into arterial cells ${ }^{52}$. However, Nr2f2 expression was not altered by Slit2 overexpression in heart cultures; therefore, it appears the impact of Slit2 on EC identity is only partial, suggesting additional factors are required for normal EC maturation. Therefore, future studies should interrogate the combinatorial paracrine code that governs normal AV specification.

Importantly, acquired and developmental vascular abnormalities underlie many human diseases, including stroke and heart disease. For example, coronary artery disease (CAD) disrupts the vascular network that supplies the heart with oxygen and 
a

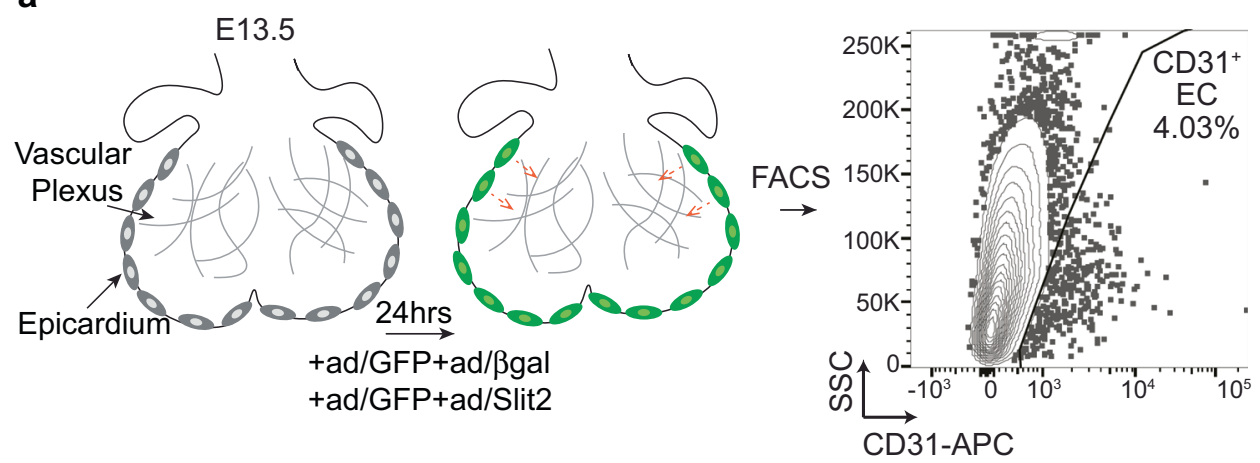

b
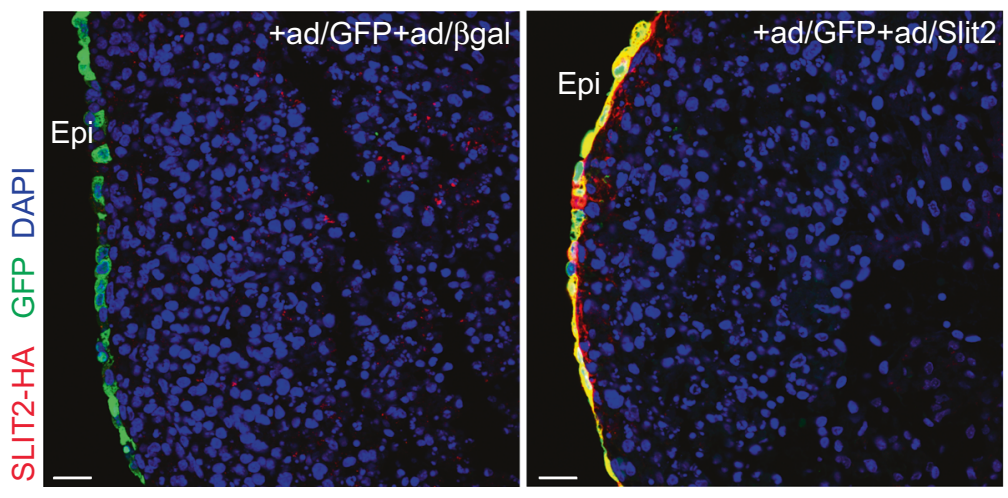

C

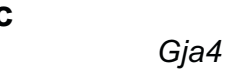

d
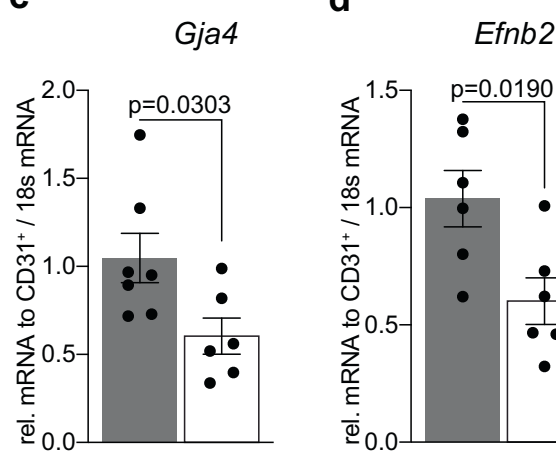

e
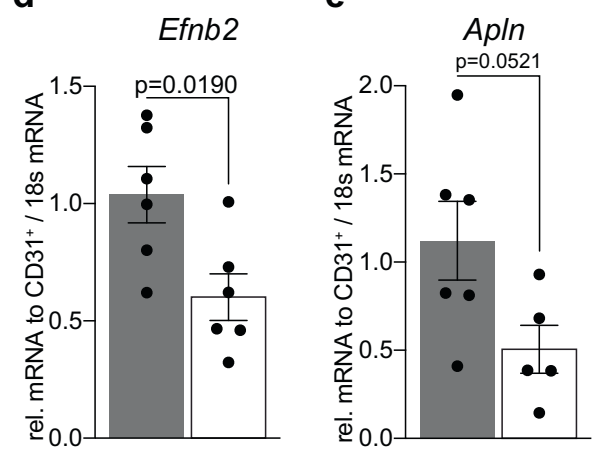

$\mathbf{f}$
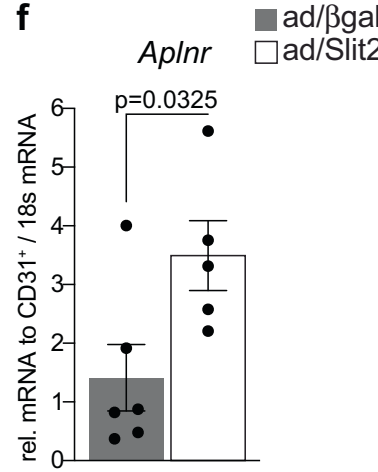

Fig. 8 SLIT2 expression in the epicardium inhibits artery specification. a Schematic of experimental design to isolate ECs following adenovirus infection of the epicardium. Hearts were extracted at embryonic day (E) 13.5 and infected with adenovirus (ad) to express $\beta$-galactosidase (ad/ $\beta$-gal) or SLIT2-HA (ad/Slit2, red). Ad expressing GFP was added to hearts to confirm the specificity of infection to cells of the epicardium (green). Following 24-h, hearts were digested and subjected to FACS to acquire CD31 expressing ECs. Refer to Supplementary Fig. 23d, e for FACS sequential gating and enrichment of ECs. b Representative images of embryonic hearts following infection with adenoviruses. SLIT2 protein expression was detected in the epicardium using an anti-HA antibody. Scale bar, $20 \mu \mathrm{m}$. DAPI staining was utilized to visualize nuclei (blue). Immunostaining was repeated independently 3 times with similar results. c-f Gene expression represented as fold change relative to $\mathrm{CD} 31^{+}$cells acquired from ad/ $\beta$ gal-treated hearts. $n$ represents samples acquired from independent embryos. ad/ $\beta$-gal $n=6$ for Efnb2, Apln, Aplnr and $n=7$ for Gja4; and ad/Slit2 $n=5$ for Apln and Aplnr and $n=6$ for Gja4 and Efnb2. Data are presented as mean values \pm SEM. Statistical significance was determined by a two-sample unpaired student's $t$-test.

nutrients. Although environmental factors including a sedentary lifestyle and a high-fat diet contribute to CAD progression, accumulating evidence suggests a considerable genetic component to disease risk ${ }^{53}$. One of the strongest genetic risk factors for CAD is the Tcf21 gene, which is highly expressed in the fetal epicardium and is essential for normal cardiac fibroblast and coronary vessel formation ${ }^{46,47}$. Therefore, a better understanding of epicardium-directed coronary vessel formation in development may provide insight into CAD mechanisms.

Regenerative therapeutic strategies for cardiac repair include approaches to promote cardiomyocyte proliferation ${ }^{54}$ and sympathetic innervation ${ }^{55,56}$; however, strategies to stimulate re-vascularization such as through enhancing coronary collateralization must complement new muscle formation. Single-cell transcriptomic analysis has identified populations of neovasculogenic ECs that emerge following $\mathrm{MI}^{57}$, and limited angiogenesis of the injured adult heart is reported to occur through the activation of developmental angiogenic programs ${ }^{58,59}$. Indeed, the epicardium induces a fetal gene program after myocardial infarction that includes a paracrine signature 60,61 . Unfortunately, the reactivation of endogenous angiogenic programs in the adult heart is insufficient to support meaningful collateralization of ischemic tissue. Therefore, our study describing the paracrine cues underlying developmental coronary angiogenesis may provide a framework to establish revascularization strategies for cardiac regenerative medicine. 
a
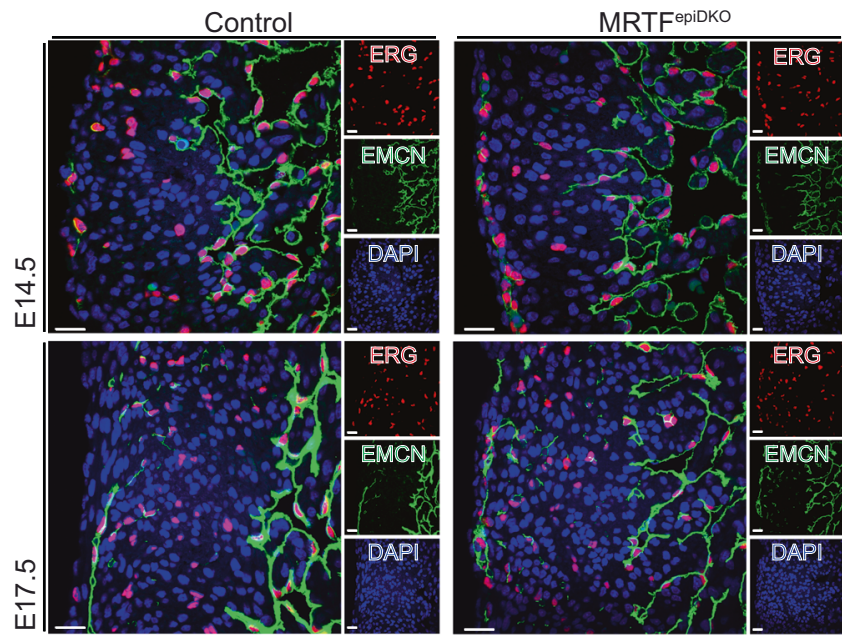

d

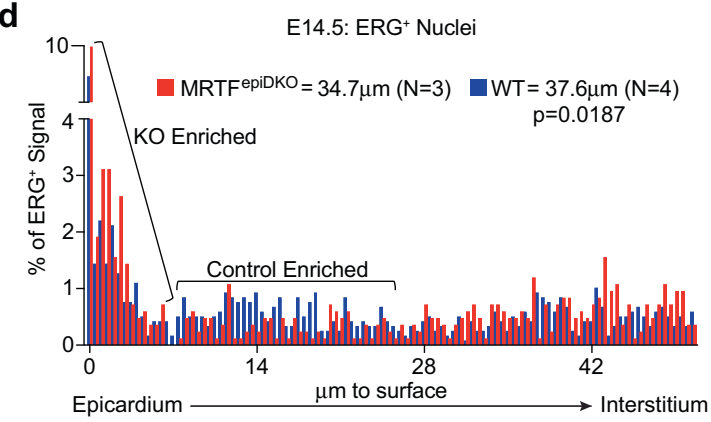

f

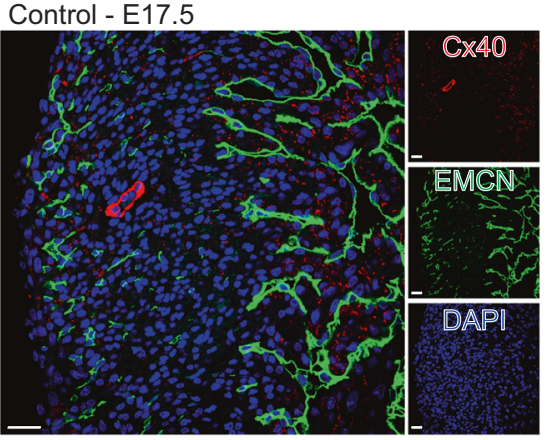

MRTFepiDKo - E17.5

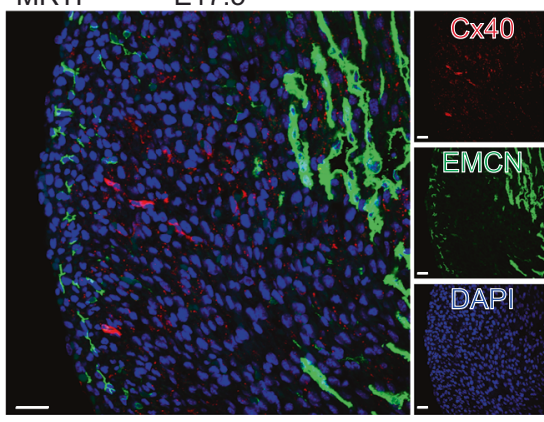

e

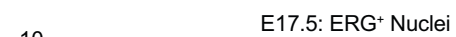

E17.5: ERG ${ }^{+}$Nuclei
ERG ${ }^{+}$Nucle

b Length to Width Ratio

1.75
$1.70-$

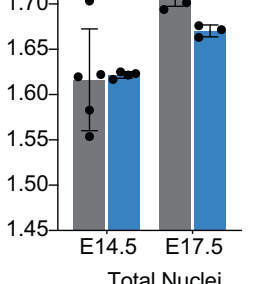

C

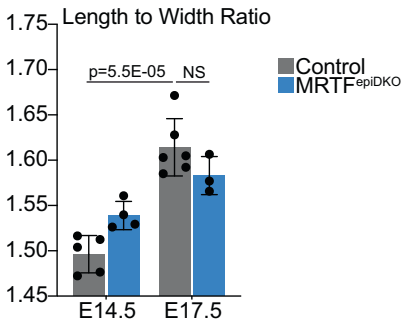

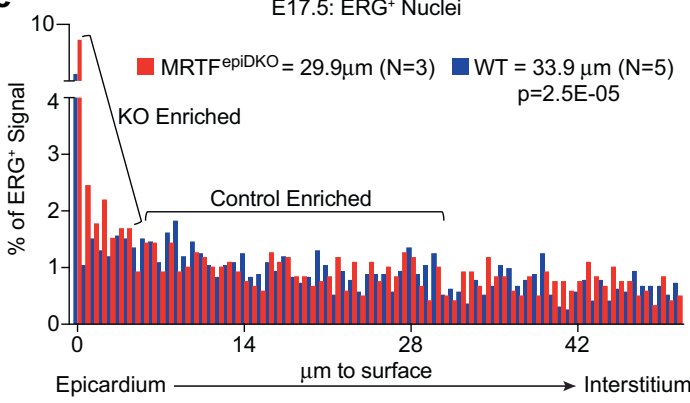

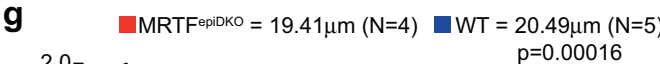

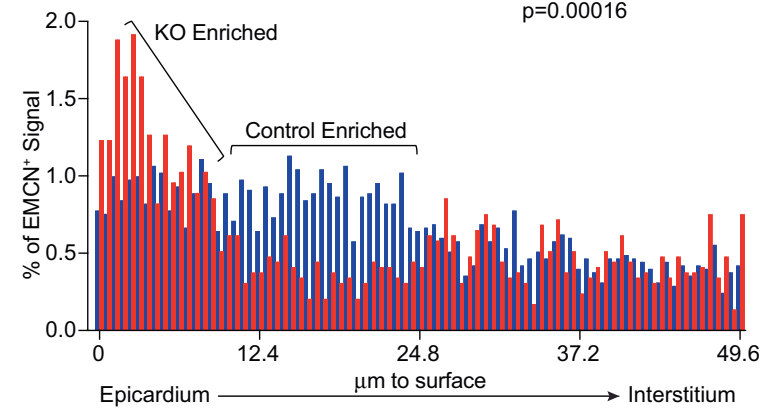

h

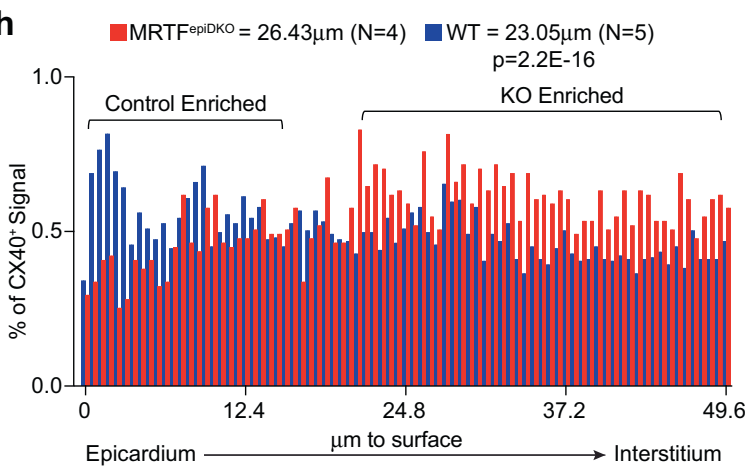

\section{Methods}

Animal models. All animal experiments were conducted in accordance with the ethical regulations for testing and research and approved by the University Committee on Animal Resources at the University of Rochester (UCAR-2011 026E). C67BL/6J mice were purchased from The Jackson Laboratory (stock number 000664) and all mouse lines were maintained on the C57BL/6J background. Rosa $26^{\mathrm{mTm}} / \mathrm{mTmG}$ mice were purchased from The Jackson Laboratory (stock number 007576). Rosa26 tdTomato mice were purchased from The Jackson Laboratory (stock number 007909). The Wt1 CreERT2 mouse strain expresses the CreERT2 fusion protein in the presence of tamoxifen and under the control of Wt1 promoter. Wt1 ${ }^{\text {CreERT2 }}$ mice were used to efficiently label the epicardium and its derivatives and as previously described ${ }^{6}$ and were purchased from The Jackson Laboratory (stock number 010912). Cspg4 CreERT2 mice express the tamoxifeninducible Cre-recombinase under the control of Cspg4 promoter. Cspg4 CreERT2 
Fig. 9 Epicardial dysfunction alters endothelial cell localization and maturation. a Immunofluorescence staining of sections from hearts isolated at embryonic stage (E)14.5 and E17.5. Antibodies are directed against ERG (red, pan-EC) and EMCN (green, venous, and endocardial EC). Scale bar, $20 \mu$ m. b Quantitation of the length-to-width ratio of $\mathrm{ERG}^{+}$nuclei (top) or $\mathbf{c}$ total nuclei (bottom). $n$ represent samples acquired from independent embryos. At E14.5, $n=5$ Control hearts and $n=4$ Mrtf-a;Mrtf- $b$ double knockout (MRTFepiDKO) hearts. At E17.5, $n=6$ Control hearts and $n=3$ MRTFepiDKO hearts. For each heart, at least three fields of view were assessed. Data are presented as mean values \pm SEM. Statistical significance was determined by a two-sample unpaired Student's $t$-test. d, e Quantitation of ERG ${ }^{+}$nuclei localization, reported as a percentage of cells within a particular bin representing the distance from the epicardial surface of the heart at $\mathbf{d}$ E14.5 and e E17.5. $\mathbf{f}$ Immunofluorescence staining of sections from hearts isolated at E17.5 with antibodies directed against EMCN (green) and Cx40 (red, arterial). Scale bar, $25 \mu \mathrm{m}$. $\mathbf{g}$, $\mathbf{h}$ Quantitation of $\mathbf{g} \mathrm{EMCN}^{+}$cell localization and $\mathbf{h} \mathrm{Cx}_{40}{ }^{+}$cell localization, reported as a percentage of cells within a particular bin representing the distance from the epicardial surface of the heart. For localization experiments, $n$ represents data acquired from independent embryos, which was analyzed in 1 experiment. For ERG + nucleus localization $n=4$ Control hearts and $n=3$ MRTFepiDKO hearts at E14.5; and $n=5$ Control hearts and $n=4$ MRTFepiDKO hearts at E17.5. For Cx40 and Emcn localization, $n=5$ Control hearts and $n=$ 4 MRTFepiDKO hearts at E17.5. Significant accumulation of ECs in particular regions of the heart are marked by brackets that indicate the over-represented genotype. For each heart, at least three fields of view were assessed. DAPI staining was utilized to visualize nuclei (blue). For data in $\mathbf{d}, \mathbf{e}, \mathbf{g}$, $\mathbf{h}$ statistical significance was determined by a two-tailed Mann-Whitney test. NS not-significant, WT wild-type, KO knockout.

mice were used to label cardiac pericytes during embryonic development and is a validated model to label Cspg4 expressing cells ${ }^{35}$ and were purchased from The Jackson Laboratory (stock number 008538). $\mathrm{Mrtfa}^{-1-}$ and $\mathrm{Mrtfb}^{\text {flox/flox }}$ mice were previously described ${ }^{7}$ and were gifts from Dr. Eric Olson (UT Southwestern, Dallas, TX, USA). The Srfflox/flox mice were previously described ${ }^{62}$ and were a gift from Dr. Joseph Miano (Augusta University, Augusta, GA, USA).

Timed pregnancies were determined after placing one male with up to two females in a single cage in the late afternoon. The next morning, a confirmed plug was termed as embryonic day (E) 0.5. In order to induce Cre-based recombination, 4-Hydroxytamoxifen (4-OHT, Millipore Sigma H6278) was dissolved in sunflower seed oil from helianthus annus (Millipore Sigma S5007) at a final concentration of $10 \mathrm{mg} / \mathrm{mL}$ with $10 \%$ ethanol. $4-\mathrm{OHT}$ was administered by oral gavage at $75 \mathrm{mg} / \mathrm{kg}$ to pregnant dams.

4-OHT administration and dissection schedules for individual experiments were: (1) The breeding strategy to generate developmentally staged embryos for single-cell RNA-sequencing of epicardial cells and isolation of hearts for immunostaining and in situ hybridization assays: Wt1 CreERT2/+ males were crossed to Rosa ${ }^{\mathrm{mTmG} / \mathrm{mTmG}}$ females. 4-OHT was administered at E9.5 and E10.5 and embryos were isolated at E12.5 and E16.5. (2) The breeding strategy to generate developmentally staged embryos for gene expression analysis in epicardial cells: Wt1 ${ }^{\mathrm{CreERT} 2 /+}$ males to $\mathrm{Rosa}^{\mathrm{mTm} / \mathrm{mTmG}}$ females or Rosa ${ }^{\text {tdTomato/tdTomato }}$ females. 4-OHT was administered at E9.5 and E10.5 and embryos were isolated at E12.5, E14.5, and E16.5. (3) The breeding strategy to generate developmentally staged embryos for the analysis of cardiac pericytes by in situ hybridization assays: Cspg 4 CreERT2/+ males were crossed to Rosa ${ }^{\mathrm{mTmG}} / \mathrm{mTmG}$ females. 4-OHT was administered at E9.5/E10.5 and E15.5/E16.5 and embryos were isolated at E17.5. (4) The breeding strategy to generate developmentally staged embryos for single-cell RNA-sequencing of endothelial cells and isolation of hearts for immunostaining and in situ hybridization assays: $\mathrm{Wt1}$ CreERT2/+ males were crossed to C57BL/6J mice to generate Control embryos. Wt1 ${ }^{\text {CreERT2/+}}$; Mrtf-a $^{-l-}$; Mrtf-b $\mathrm{b}^{\text {flox/flox }}$ males were crossed to $\mathrm{Mrtf}^{-\mathrm{a}^{-l-}}$; Mrtf-b $\mathrm{b}^{\text {flox/flox }}$ females to generate MRTFepiDKO embryos. 4-OHT was administered at E9.5 and E10.5 and embryos were isolated at E14.5 and E17.5. (5) The breeding strategy to generate developmentally staged embryos for isolation of Control and MRTF mutant epicardial cells for bulk RNA-sequencing and gene expression studies: Mrtf-a $^{-1-}$; Mrtf-b $\mathrm{b}^{\text {flox/flox }}$ males were crossed to Mrtf-a ${ }^{-/-}$; Mrtf-b $\mathrm{b}^{\text {flox/flox }}$ to generate Mrtf-a $^{-1-} ;$ Mrtf-b $\mathrm{b}^{\text {flox/flox }}$ embryos. SRFflox/flox males were crossed to SRFflox/flox females to generate SRFflox/flox embryos. Embryos were dissected at E12.5 for heart culture and epicardium-derived cell labeling and gene deletion was conducted via adenoviral-vector mediated delivery of GFP and Cre-recombinase, as described below. (6) The breeding strategy to generate developmentally staged embryos for ex vivo expansion of primary epicardial cells and gene expression studies: C57BL/6 males were crossed to C57BL/6J females and embryos were isolated at E11.5. (7) The breeding strategy to generate developmentally staged embryos for isolation of endothelial cells following ex vivo heart culture and infection with adenoviruses: C57BL/6J males were crossed to C57BL/6J females and embryos were isolated at E13.5.

Embryonic heart digestion protocol. Epicardium-derived cells (EPDCs) and endothelial cells (ECs) were isolated from developmentally staged hearts as defined above. On the day of isolation, pregnant dams were anesthetized with an intraperitoneal injection of $0.5 \mathrm{~mL}$ of ketamine-xylazine cocktail $(13 \mathrm{mg} / \mathrm{mL}$ ketamine in $0.88 \mathrm{mg} / \mathrm{mL}$ xylazine in DPBS) followed by cervical dislocation. After the use of $70 \%$ ethanol to sterilize the abdominal area, an incision to enter and remove decidua away from the mesometrium was performed, and embryos were placed in pre-warmed HBSS (ThermoFisher Scientific, SH30031.02). After the removal of extraembryonic tissue and the yolk sac, the heart was removed from the embryo and placed in a cell culture well-containing culture media made up of M199 (ThermoFisher Scientific, SH3025301) supplemented with 10\% FBS (Gemini BioProducts, 100106) and 1\% Penicillin/Streptomycin (Pen-Strep; ThermoFisher Scientific, SV30010). Digestion of embryonic hearts began by removing residual
HBSS from wells and replacing media with a digestion solution containing $0.08 \%$ Collagenase IV (Millipore Sigma, C5138), 0.05\% Trypsin Protease (ThermoFisher Scientific, SH30042.01), 1\% chicken serum (Vector Laboratories, S-3000) diluted in pre-warmed HBSS before placing hearts in a $37^{\circ} \mathrm{C}$ hybridization oven with gentle shaking for $5 \mathrm{~min}$ intervals. Following incubation, hearts were dissociated by gentle pipetting ( 3 times with a P1000 pipette) and undigested tissue was allowed to settle for $30 \mathrm{~s}$. After settlement of tissue, media was collected and added to a separate tube containing horse serum (Vector Laboratories, S-2000) to neutralize digestion, and digested cells were then saved on ice. Digestion, pipetting, and collection of media were repeated 3-5 more times, and cells were then filtered through a $70 \mu \mathrm{m}$ filter and centrifuged at $200 \times g$ for $5 \mathrm{~min}$ at $4{ }^{\circ} \mathrm{C}$. The resulting pellet was placed in $10 \%$ FBS in DMEM (without phenol red, ThermoFisher Scientific, SH30284.01) and saved on ice before performing fluorescence-activated cell sorting FACS using a BD FACS Aria II using a $100 \mu \mathrm{m}$ nozzle (BD Biosciences). DAPI (4',6-Diamidino-2-Phenylindole, Dihydrochloride) was added to cells immediately before sorting $(0.5 \mu \mathrm{g} / \mathrm{mL}$; ThermoFisher Scientific, D1306) to exclude dead cells. Cells were sorted directly into $1.5 \mathrm{~mL}$ Eppendorf tubes containing $0.5 \%$ bovine serum albumin (BSA, Millipore Sigma, A9647) in DPBS at $4{ }^{\circ} \mathrm{C}$ and immediately processed.

Cell isolation of epicardial cells at E12.5 and E16.5 for scRNA-seq. EPDCs were collected from Wt1 ${ }^{\mathrm{CreERT} 2 /+}$; R26 $6^{\mathrm{mTmG} /+}$ embryos that were administered 4-OHT at E9.5 and E10.5 via pregnant dams. A total of 7 E12.5 staged hearts were pooled from 2 dams, and a total of 17 E16.5 staged hearts were pooled from 4 dams based on visual confirmation of green fluorescent protein (GFP) expression in the epicardium using a ZOE Fluorescent Cell Imager (Bio-Rad). Hearts negative for the expression of the $\mathrm{Wt1}{ }^{\mathrm{CreERT} 2}$ allele, exhibited tdTomato fluorescence alone, and were either discarded or used as tdTomato positive fluorescence controls for flow cytometry. Developmentally staged C57BL/6J embryos were collected as nonfluorescence controls for flow cytometry. Additionally, genomic DNA was isolated from all embryos, and $\mathrm{Wt} 1$ CreERT2; $\mathrm{R} 26^{\mathrm{mTmG} /+}$ positive embryos were confirmed by PCR genotyping using transgene-specific primers. Following the digestion protocol described, EPDCs were gated as single cells (based on FSC $\times$ SSC dimensions), DAPI negative, tdTomato negative, and GFP-positive. TdTomato positive cells were sorted for downstream gene expression analysis. EPDCs collected by FACS were immediately processed for single-cell capture, library preparation, and sequencing, as described below.

Cell isolation of epicardial cells at E12.5, E14.5, and E16.5 for gene expression analysis. EPDCs were collected from both $\mathrm{Wt} 1^{\mathrm{CreERT} 2 /+} ; \mathrm{R} 26^{\mathrm{mTmG} /+}$ and $\mathrm{Wt1}^{\mathrm{CreERT} 2 /+}$; R26 $6^{\mathrm{tdT} T o m a t o /+}$ embryos that were administered 4-OHT at E9.5 and E10.5 via pregnant dams. Fluorescence was confirmed using the ZOE Fluorescent Cell Imager (Bio-Rad). Hearts negative for the expression of the Wt1 ${ }^{\text {CreERT2 }}$ allele, exhibited tdTomato fluorescence $\left(\mathrm{R} 26^{\mathrm{mTmG} /+}\right)$ or were non-fluorescent $\left(\mathrm{R} 26^{\text {tdTomato/+ }}\right)$ and were either discarded or used as fluorescence controls for flow cytometry. Following the digestion protocol described, EPDCs were gated as single cells (based on FSC $\times$ SSC dimensions), DAPI negative, tdTomato negative, and GFP-positive if the cross was to the $\mathrm{R} 26^{\mathrm{mTmG}}$ fluorescent reporter. If the R26 ${ }^{\text {tdTomato }}$ fluorescent reporter was used, DAPI negative and tdTomato positive EPDCs were collected. EPDCs collected by FACS were then processed for RNA isolation prior to conducting quantitative RT-PCR.

Cell isolation of endothelial cells at E14.5 for scRNA-seq. ECs were collected

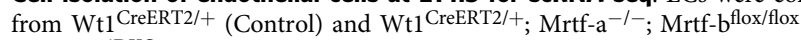
(MRTFepiDKO) mice after administration of 4-OHT at E9.5 and E10.5 via oral gavage of pregnant dams. A total of 10 Control hearts were pooled from 2 dams. A total of 7 MRTFepiDKO hearts were pooled from 2 dams. Prior to digestion, hearts were placed in $\mathrm{HBSS}$ at $37^{\circ} \mathrm{C}$ and $5 \% \mathrm{CO}_{2}$ and genomic DNA from all embryos were subjected to genotyping to detect the $\mathrm{Wt1}^{\mathrm{CreERT} 2 /+}$ allele within $2 \mathrm{~h}$. Following confirmation of positive embryos, hearts were subjected to the digestion 
protocol described. After filtering and centrifuging cells, ECs were incubated with fluorescently conjugated antibodies directed against CD31-APC (dilution at 1:100; BD Biosciences 551262) and CD45-FITC (dilution at 1:50; BD Biosciences 553079) for $30 \mathrm{~min}$ in $0.5 \%$ BSA in DPBS on ice. After antibody labeling, cells were washed and centrifuged at $200 \mathrm{~g}$ for $5 \mathrm{~min}$ and placed in $10 \% \mathrm{FBS} / \mathrm{DMEM}$ buffer. ECs were gated as single cells that are DAPI negative, CD45-FITC negative, and CD31-APC positive. ECs collected by FACS were immediately processed for single-cell capture, library preparation, and sequencing.

\section{Ex vivo embryonic heart culture for isolation of endothelial cells following}

adenovirus infection. ECs were collected from C57BL/6 hearts that were extracted at E13.5 and placed in culture media (DMEM:M199 with 10\% FBS and 1\% PenStrep) containing adenovirus to express $\beta$-galactosidase (Vector Biolabs, 1080) or SLIT2-HA (Applied Biological Materials, 132844A) for $24 \mathrm{~h}$ at $37^{\circ} \mathrm{C}$ and $5 \% \mathrm{CO}_{2}$ and subjected to the digestion protocol described. This method primarily transduces surface epicardial cells with adenovirus. After filtering and centrifuging cells, ECs were incubated with fluorescently conjugated antibodies to select for vascular EC (CD31-APC; BD Biosciences 551262) for $30 \mathrm{~min}$ in 0.5\% BSA in DPBS on ice. After antibody labeling, cells were washed and centrifuged at $200 \times g$ for $5 \mathrm{~min}$ and placed in 10\%FBS/DMEM buffer. ECs were gated as single cells that are DAPI negative and CD31-APC positive. ECs collected by FACS were immediately processed for RNA isolation prior to conducting quantitative RT-PCR.

\section{Ex vivo embryonic heart culture for isolation of epicardial cells for bulk RNA-} sequencing. Hearts were collected from Srf flox/flox (for control EPDC, Srf-KO EPDCs, and non-EPDC) and Mrtf-a ${ }^{-1-}$; Mrtf-b flox/flox (for Mrtf-dKO) embryos that were extracted at E12.5 and placed in culture media (M199 with 10\% FBS and $1 \%$ Pen-Strep) containing TGF- $\beta 2$ (2 ng/mL; R\&D Systems) and PDGF-BB (20 ng/mL; R\&D Systems) to induce epithelial-mesenchymal transition. All explants were transduced with adenovirus to express a green fluorescent protein (GFP, Vector Biolabs, 1060) on the epicardial surface. Control hearts were cotransduced with adenovirus expressing $\beta$-galactosidase (Vector Biolabs, 1080) while gene deletion was accomplished by co-transduction with adenovirus expressing Cre-recombinase (Vector Biolabs, 1045) to excise floxed alleles (all adenovirus treatments were at $1 \times 10^{6} \mathrm{pfu} / \mathrm{mL}$ ). Following $48 \mathrm{~h}$ of culture at $37^{\circ} \mathrm{C}$ and $5 \% \mathrm{CO}_{2}$, hearts were dissociated and EPDCs were isolated via FACS by gating for single cells, and separated as GFP negative (non-EPDCs) or GFP-positive (EPDCs) from each group and collected in $5 \mathrm{~mL}$ FACS tubes containing $0.5 \mathrm{~mL}$ HBSS supplemented with $10 \%$ FBS. Hearts not treated with ad-GFP were used as non-fluorescence gating controls during flow cytometry analysis. Sorted cells were then pelleted at $200 \times g$ for $5 \mathrm{~min}$ at $4^{\circ} \mathrm{C}$. Total RNA was isolated using TRIzol Reagent (ThermoFisher Scientific, 15596018) per manufacturer's instructions and cleaned up with column purification. RNA quality was evaluated using a bioanalyzer and prepared into NGS libraries for bulk RNA-sequencing or was used for conducting quantitative RT-PCR.

\section{Single library preparation and processing of single epicardial cells and} endothelial cells. Single-cell libraries were generated from epicardial cells and endothelial cells acquired by FACS. Prior to capture using the $10 \times$ Genomics Chromium controller (10x Genomics), the number of cells was quantitated (TC20 Automated Cell Counter, Bio-Rad) and cell viability was assessed via the trypan blue exclusion test of cell viability. Only cell populations exhibiting greater than $80 \%$ viability were used. All cells were loaded in order to maximize the number of single cells acquired using the Chromium single Cell $3^{\prime}$ Reagent Kit. Libraries were prepared according to the manufacturer's instructions using the Chromium Single Cell $3^{\prime}$ Library and Gel Bead Kit v.2 (10× Genomics). CellRanger v2.2.0 was used to demultiplex each capture, process base-call files to fastq format, and perform $3^{\prime}$ gene counting for each individual cell barcode with mouse reference data set $(\mathrm{mm10}, \mathrm{v} 2.1 .0)$.

Single-cell transcriptome sequencing of epicardial cells. Cell filtering and celltype annotation and clustering analysis: Quality control, identification of variable genes, principle component analysis, and non-linear reduction using UMAP were performed using Seurat (v3.0.0.9000 and R v3.5.1) for each individual time point separately. The integration function RunCCA was utilized to identify cell typespecific clusters without respect to developmental time. Cell-type annotations were identified based on significant cluster-specific marker genes and the Mouse Gene Atlas using Enrichr (enrichR_2.1). In order to understand the effect of developmental time, the Seurat (v3.0.0.9150) function merge() was used to combine the E12.5 and E16.5 captures to maintain the variation introduced by developmental time. Cell cycle scoring was performed and the variation introduced as a number of genes involved in mitochondrial transcription, and cell cycle phases S and G2/M were regressed out during data scaling. Data was visualized in UMAP space and clustered were defined using a resolution of 0.5 .

Developmental trajectory and prediction of cell-fate determinants: The GetAssayData() function in Seurat (v3.0.0.9150) was used to extract the raw counts to construct the Monocle object. To construct the trajectory the default functions and parameters as suggested by Monocle (v2.10.1) were used along with the following deviations: the hypervariable genes defined using Seurat VariableFeatures
() function were used as the ordering genes in Monocle, 8 principle components were used for further non-linear reduction using tSNE, and num_clusters was set to 5 in the clusterCells() Monocle function. The resulting Monocle trajectory was colored based on Monocle State, Pseudotime, developmental origin (E12.5 or E16.5), and Seurat clusters previously identified. Genes that are dynamically expressed at the one identified branchpoint were analyzed using the BEAM() function. The top 50 genes that are differentially expressed at the branchpoint were visualized using the plot_genes_branched_heatmap() function in Monocle.

Integration with Mouse Cell Atlas. Neonatal hearts from one-day-old pups were downloaded from the Mouse Cell Atlas (https://figshare.com/articles/ MCA_DGE_Data/5435866) and re-analyzed using Seurat v3 following standard procedures previously outlined. Epicardial (E12.5 and E16.5) and neonatal-heart (1 day old) were integrated using the FindIntegegrationAnchors() and IntegrateData() functions using Seurat v3. Data were visualized in the 2dimensional UMAP space. Marker genes were identified for the integrated clusters and Enrichr (enrichR_2.1) was used to identified significantly enriched Biological Processes (Gene Ontology 2018).

Single-cell transcriptome sequencing of endothelial cells. Cell filtering, celltype clustering analysis, and creation of cellular trajectories: Seurat (3.0.2) was used to filter low-quality cells, score the cells by the cell cycle, and integrate the E14.5 MRTFepiDKO and Control datasets using the merge function. Cells were clustered using the first 36 dimensions of PCA to the resolution of 0.7 and visualized using UMAP. Monocle (2.10.1) was used to infer cellular trajectory after the removal of cell cycle-related genes. The determined cell states were used to determine cell state proportions of MRTFepiDKO and Control and identify potential markers for these cell states. Originating datasets, pseudotime states, and cell cycle state colorings were used within generated graphics.

Receptor-ligand expression analysis: Using published lists of pairings from Ramilowski et al. ${ }^{63}$, the receptor-ligand pairings were converted to MGI gene symbol from HGNC gene symbol using biomaRt $(2.42 .0)^{64,65}$. Ligands that were shown to be differentially expressed within the whole-transcriptome sequencing of the MRTFepiDKO epicardial cells in comparison to the Control were flagged for later consideration.

Both the endothelial and epicardial datasets were filtered for expressed receptors and ligands, respectively. Ligands expressed within the epicardial data set were categorized as being differentially expressed between mesothelial and mesenchymal cell populations. Receptors expressed within the E14.5 MRTFepiDKO and Control combined data set were characterized as differentially expressed between the two conditions. Seurat's DotPlot and doHeatMap functions were used to visualize differential expression across both datasets.

For network visualization, tidyverse $(1.3)^{66}$ was used for data analysis, viridis (0.5.1) (https://cran.r-project.org/web/packages/viridis/index.html) was used for color mapping, and both igraph (1.2.4.2) (https://igraph.org/) and ggraph (2.0.1) (https://cran.r-project.org/web/packages/ggraph/index.html) were used to generate and plot the network map. Epicardial ligands and endothelial receptors were grouped together and colored based on differential regulation; green if they were solely differentially regulated within that data set or red if they had a corresponding differentially regulated ligand or receptor. Red-lines connect receptors and ligand pairs, which were both confirmed to be differentially expressed. The epicardial ligands were further colored by expression in specific cell populations identified as mesothelial, mesenchymal, or general epicardial.

Whole-transcriptome sequencing of epicardial cells. The Clontech Ultralow RNA Kit in conjunction with NexteraXT DNA Library Prep Kit (Illumina) was used for next-generation sequencing library construction according to the manufacturer's protocols. Briefly, mRNA was purified from $1 \mathrm{ng}$ total RNA with oligo$\mathrm{dT}$ magnetic beads and fragmented. First-strand cDNA synthesis was performed with random hexamer priming followed by second-strand cDNA synthesis using dUTP incorporation for strand marking. End repair and $3^{\prime}$ adenylation was then performed on the double stranded cDNA. Illumina adaptors were ligated to both ends of the cDNA, purified using Ampure beads, and amplified with PCR primers specific to the adaptor sequences to generate cDNA amplicons of $\sim 200-500 \mathrm{bp}$ in size. The amplified libraries were hybridized to the Illumina single-end flow cell and amplified using the cBot (Illumina). Single-end reads of 100 nt were generated for each sample using Illumina's HiSeq2500v4. Raw reads were generated from Illumina HiSeq2500 sequencing and demultiplexed using bcl2fastq version 1.8.4. Quality filtering and adapter removal were performed using Trimmomatic version 0.32 with the following parameters: "TRAILING:13 LEADING:13 ILLUMINACLIP:adapters.fasta:2:30:10 SLIDINGWINDOW:4:20 MINLEN:15". Processed/ cleaned reads were then mapped to the GRCm38 reference genome using the SHRiMP version 2.2.3 and the following parameters: "-qv-offset 33-all-contigs". Uniquely aligned and multi-mapped reads were counted within the gencode GRCm38 gene annotations, in a strand-specific manner, using the cuffdiff tool from the cufflinks-2.0.2 package and the following parameters: "-FDR $0.05-\mathrm{u}-\mathrm{b}$ GENOME". Differential expression analyses and data normalization were performed using DESeq2-1.14.1R/Bioconductor package with an adjusted $p$-value (Benjamini-Hochberg) threshold of 0.05 within the R version 3.3.1 environment (https://www.R-project.org). The PCA plot was created given the top500 genes with the most variation using the stats-3.4.0 (prcomp) and rgl-0.98.1R packages. 
$K$-means clustering was performed on DEGs using log-transformed, normalized counts in Cluster 3.0 (http://bonsai.hgc.jp/ mdehoon/software/cluster/software. $\mathrm{htm}$ ). Heat maps were generated using TreeView software (Version 1.1.6r4) and GraphPad (Version 8.4.2). Gene ontology analysis was performed using EnrichR and Ingenuity Pathway Analysis. Kyoto Encyclopedia of Genes and Genomes (KEGG) pathway analysis was performed using EnrichR.

Epicardial explant culture and induction of EMT. In order to acquire primary epicardial cells for in vitro experiments, pregnancies were timed to obtain E11.5 embryos from C57BL/6J female dams ${ }^{34}$. On the day of isolation, pregnant dams were anesthetized and administered ketamine-xylazine via intraperitoneal injection followed by cervical dislocation. After removal of decidua, embryos were placed in pre-warmed HBSS, extraembryonic tissue and the yolk sac were dissected, and hearts were extracted from the embryo and placed dorsal side down on collagencoated culture wells (Corning, 354557) and incubated at $37^{\circ} \mathrm{C}$ and $5 \% \mathrm{CO}_{2}$ for 30 min to allow adhesion of hearts to the collagen matrix. Following incubation, media composed of M199 supplemented with 5\% FBS and 1\% Pen-Strep was added slowly around the hearts $(50-100 \mu \mathrm{L})$ and incubated at $37^{\circ} \mathrm{C}$ and $5 \% \mathrm{CO}_{2}$ for approximately $24 \mathrm{~h}$ to allow for epicardial outgrowth. Next day, hearts were removed using fine-tip forceps and the epicardial cell monolayer was washed 2 times with DPBS. Primary epicardial cells were then treated with culture media (M199 with 1\% FBS and 1\% Pen-Strep) containing recombinant human TGF- $\beta 1$ $(10 \mathrm{ng} / \mathrm{mL})$ and recombinant human PDGF-BB $(20 \mathrm{ng} / \mathrm{mL})$ to induce EMT for a total of $48 \mathrm{~h}$ (with replenishment of fresh media and recombinant factors after 24 h) at $37^{\circ} \mathrm{C}$ and $5 \% \mathrm{CO}_{2}$. After a total of $72 \mathrm{~h}$ in culture, epicardial cells were lysed in TRIzol Reagent and processed for RNA isolation. Gene expression analysis was performed with samples combined from two separate experiments. Vehicle $(n=$ $6-7)$ and TGF $\beta 1 /$ PDGF-BB $(n=7-9)$.

RNA isolation, cDNA biosynthesis, and quantitative RT-PCR. RNA was isolated using TRIzol Reagent according to the manufacturer's instructions. RNA was treated with the TURBO DNA-free Kit (ThermoFisher Scientific, AM1907) to eliminate genomic DNA. Purified RNA was then made into cDNA using iScript cDNA Synthesis Kit (Bio-Rad, 1708891BUN). qRT-PCR was performed with cDNA, primers (Supplementary Table 1), and IQ SYBR Green Supermix (Bio-Rad, 1708887). Data were analyzed using the $\Delta \Delta C(t)$ method. Samples with insufficient melt curves were not used in analyses. Due to the low yields of RNA following enrichment or culture of embryonic cardiac cells, varying $n$ displayed in Figs. 4, 5, and 8 and Supplementary Fig. 23 are reflective of samples that could not be included in all gene expression analyses due to insufficient cDNA quantities.

In situ hybridization assays. Embryonic hearts from $\mathrm{Wt} 1 \mathrm{CreERT} /+$; $\mathrm{R} 26^{\mathrm{mTmG} /+}$ (E12.5 and E16.5), Cspg4 $4^{\mathrm{CreERT} 2 /+} ; \mathrm{R}_{2} 6^{\mathrm{mTmG} /+}$ (E17.5), Wt1 ${ }^{\mathrm{CreERT} 2 /+}$ (E14.5), and Wt1 ${ }^{\mathrm{CreERT} 2 /+}$; Mrtf-a ${ }^{-l-}$; Mrtf-b $\mathrm{b}^{\text {flox/flox }}$ (E14.5) were harvested and fixed in $10 \%$ neutral buffered formalin (NBF; ThermoFisher Scientific, 22-110-869) in DPBS for 18-24 hs at room temperature on a rocking platform. After fixation, tissue was dehydrated in an ethanol series followed by xylene before embedding tissue in paraffin wax and cutting hearts into $5 \mu \mathrm{m}$ sections using a microtome. After sectioning, slides were allowed to dry overnight at room temperature and stored with desiccants for long-term storage. In order to perform in situ hybridization, we utilized the RNAscope Multiplex Fluorescent V2 Assay (Advanced Cell Diagnostics, 323100) as per the manufacturer's instructions for formalin-fixed paraffin-embedded (FFPE) tissue, and with small modifications. Manual antigen retrieval was performed for $10 \mathrm{~min}$ and RNAscope protease plus solution was added for $30 \mathrm{~min}$ to each section. Following pre-treatment protocols, a combination of 2-3 mRNA probes (Supplementary Table 2) was hybridized for $2 \mathrm{~h}$ at $40^{\circ} \mathrm{C}$ and stored at room temperature in $5 \times$ Saline Sodium Citrate (SSC) overnight (16-18 h). The next day, amplification of probes was performed by hybridization and the development of horseradish peroxidase (HRP) to $\mathrm{C} 1, \mathrm{C} 2$, or $\mathrm{C} 3$ conjugated probes followed by addition of Tyramide-Signal Amplification (TSA Plus Fluorescein, Cyanine 3 and Cyanine 5; Perkin Elmer, NEL741001KT, NEL744001KT, and NEL745001KT) to fluorescently label probes (Supplementary Table 2) in a sequential manner. DAPI was added to sections after the last wash step for $30 \mathrm{~s}$, and slides were mounted with ProLong Gold Antifade Mountant (ThermoFisher Scientific, P10144), coverslipped (\#1.5 mm), and imaged using an Olympus Confocal Microscope IX81 (Olympus Corporation).

Immunohistochemistry. Embryonic hearts from Wt1 $\mathrm{CreERT} /+$; $226^{\mathrm{mTmG} /+}$

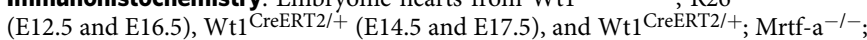
Mrtf-b flox/flox (E14.5 and E17.5) were harvested and fixed in $4 \%$ paraformaldehyde (PFA; Electron Microscopy Sciences 15710) in DPBS for 18-24 h at room temperature. After fixation, tissue was dehydrated in an ethanol series followed by xylene before embedding in paraffin wax. Hearts were then cut at $5 \mu \mathrm{m}$ sections using a microtome, baked at $60^{\circ} \mathrm{C}$ overnight, and stored at room temperature for long-term storage. To begin immunostaining, slides were deparaffinized in a series of xylenes, followed by 3 -min incubations in 100\% ethanol (EtOH, 3 times), $95 \%$ $\mathrm{EtOH}$ (1 time), and then placed in distilled water. Antigen retrieval was performed in pH6 Dako Target Antigen Retrieval buffer (Agilent Technologies, S169984-2) followed by an incubation with $3 \% \mathrm{H}_{2} \mathrm{O}_{2}$ in $15 \mathrm{mM} \mathrm{NaCl} / 100 \mathrm{mM}$ Tris pH 7.5 (TN buffer) to quench endogenous HRP. To prevent non-specific binding of antibodies, TSA Blocking Reagent (Perkin Elmer, FP1012) diluted at $0.5 \%$ in TN wash buffer (TNB) was added for at least $30 \mathrm{~min}$ to sections at room temperature. After the blocking step, primary antibodies diluted in TNB were incubated on sections overnight at $4{ }^{\circ} \mathrm{C}$. After overnight incubation (16-18 h), slides were washed 3 times in TN buffer and incubated in secondary antibodies diluted in TNB for $2 \mathrm{~h}$ at room temperature. Amplification was performed as necessary. Biotin secondaries were amplified by adding a streptavidin-conjugated fluorophore for an additional $1 \mathrm{~h}$ at room temperature in TNB after performing 3 washes of biotin secondary. HRP secondaries were amplified by using the TSA Plus Fluorescent system for $10 \mathrm{~min}$ at room temperature in amplification diluent (provided in TSA Plus kit) after performing 3 washes of an HRP secondary. Following, secondary or tertiary incubations, slides were washed 3 more times in TN buffer with the final wash containing DAPI $(0.5 \mu \mathrm{g} / \mathrm{mL})$ for at least $10 \mathrm{~min}$ to stain nuclei. Slides were mounted with VECTASHIELD Anti-Face Mounting Media (Vector Labs, H-1000) before being imaged on an Olympus Confocal Microscope IX81 (Olympus Corporation). Primary Antibodies and dilutions: Rabbit anti-GFP (1:200, Torrey Pines Biolabs Inc., TP401), Goat anti-PDGFR $\alpha$ (1:100, R\&D Systems, AF1062), Mouse anti-MYL2 (1:50, Santa Cruz Biotechnology sc517244), Mouse anti-cTNT (1:100, ThermoFisher Scientific, PIMA512960), Rabbit anti-ERG (1:200, Abcam, ab92513), Rat anti-EMCN (1:100, eBioscience, 14-5851-85), Rabbit anti-Cx40 (1:100, Alpha Diagnostic, CX40A), Rabbit anti-HA-Tag (1:100, Cell Signaling Technology, 3724s). Secondary Antibodies and dilutions: Donkey anti-Rabbit Biotin (1:500, Jackson ImmunoResearch Laboratories, 711-065-152), Bovine anti-Goat HRP (1:200, Jackson ImmunoResearch Laboratories, 805-035-180), Donkey anti-Rabbit HRP (1:200, Jackson ImmunoResearch Laboratories 711-035-152), Streptavidin-555 (1:100, ThermoFisher Scientific, S21381), Tyramide FITC (1:200, Perkin Elmer, NEL741E001KT), Tyramide Cy3 (1:200, Perkin Elmer, NEL744E001KT), Donkey anti-Mouse 647 (1:200, Jackson ImmunoResearch Laboratories, 715-605-151), Donkey anti-Rat 488 (1:200, Jackson ImmunoResearch Laboratories, 703-545-155), Donkey anti-Rabbit 488 (1:100, Jackson ImmunoResearch Laboratories, 711-545-152).

Quantitation of endothelial cell polarity. The evaluation of nuclear polarity in embryonic tissue was performed following immunostaining of hearts with endothelial-specific nuclear protein marker ERG, which was counterstained with DAPI to visualize nuclei. Quantitation of nuclear dimensions of $\mathrm{ERG}^{+}$nuclei and total nuclei was performed using ImageJ (Fiji Version: 2.0.0-rc-69/1.52p). Specifically, to measure EC nuclei, single scans of ERG and DAPI labeling (imaged via Confocal Microscope Olympus BX41 at $\times 60$ ) were colocalized using the Colocalization Threshold function in ImageJ software, automatically creating an image of all ERG ${ }^{+}$ and $\mathrm{DAPI}^{+}$nuclei. Subsequently, the images were filtered to a threshold to obtain a binary image that was watershed, and images were analyzed through the Analyze Particles function. Nuclear dimensions were evaluated via the Feret's Diameter function, and the nuclear length to width ratio was determined by dividing the Feret value by the minimum Feret of each cell ${ }^{67}$. At E14.5, 5 Control hearts and 3 MRTFepiDKO hearts were analyzed. At E17.5, 6 Control hearts and 3 MRTFepiDKO hearts were analyzed. For each heart, at least three fields of view were assessed.

Quantitation of endothelial cell localization. Evaluation of $\mathrm{ERG}^{+}, \mathrm{EMCN}^{+}$, and $\mathrm{Cx} 40^{+}$cell localization starting from the surface of the heart (epicardium) was performed using ImageJ software. The DAPI channel was used to delimit the epicardium layer, defined as the outer layer of nuclei. Each channel/protein was processed with a smoothing filter, adjusted for brightness and contrast, and filtered to obtain a mask. In order to minimize manual errors, an automated script was written to measure the distances of each channel/protein to the epicardium layer. The masks obtained in ImageJ provided the input for the script. The script was written in Python ${ }^{68}$ and utilized the image processing packages scikit-image ${ }^{69}$ and mahotas ${ }^{70}$. At E14.5, 4 Control hearts and 3 MRTFepiDKO hearts were analyzed. At E17.5, 5 Control hearts and 3 MRTFepiDKO hearts were analyzed for $\mathrm{ERG}^{+}$cells and 4 MRTFepiDKO hearts were analyzed for $\mathrm{EMCN}^{+}$and $\mathrm{Cx} 40^{+}$cells. For each heart, at least three fields of view were assessed.

Statistical analyses. Data were expressed as mean \pm SEM for bar graph data presented and statistical analyses were performed using unpaired two-tailed Student's $t$-test when comparing two groups. All measurements in this paper were acquired from distinct samples and no samples were measured repeatedly. Bar graph data analysis was performed using GraphPad Prism 8 for macOS (Version 8.4.2). Statistical analysis of endothelial cell localization was performed using a two-tailed Mann-Whitney test. A value of $p<0.05$ was considered statistically significant.

Reporting summary. Further information on research design is available in the Nature Research Reporting Summary linked to this article.

\section{Code availability}

All transcriptomic analyses were performed using standard protocols with previously described R packages in the methods. Analysis of endothelial cell localization was determined using Python script described in the methods. $\mathrm{R}$ and Python scripts mentioned in this manuscript are available upon request. 


\section{Data availability}

Bulk RNA-sequencing data from epicardial cells have been deposited in the Gene Expression Omnibus database under accession code "GSE153367". Single-cell transcriptomic analysis of epicardial cells and endothelial cells data generated in this study have been deposited in the Gene Expression Omnibus database under accession code "GSE154715". All other relevant data supporting the key findings of this study are available within the article and its Supplementary Information files or from the corresponding author upon reasonable request. Source data are provided with this paper.

Received: 6 August 2020; Accepted: 18 June 2021;

Published online: 06 July 2021

\section{References}

1. Red-Horse, K., Ueno, H., Weissman, I. L. \& Krasnow, M. A. Coronary arteries form by developmental reprogramming of venous cells. Nature 464, 549-553 (2010)

2. Fish, J. E. \& Wythe, J. D. The molecular regulation of arteriovenous specification and maintenance. Dev. Dyn. 244, 391-409 (2015).

3. Carmeliet, P. Mechanisms of angiogenesis and arteriogenesis. Nat. Med. 6, 389-395 (2000).

4. Giacca, M. \& Zacchigna, S. VEGF gene therapy: therapeutic angiogenesis in the clinic and beyond. Gene Ther. 19, 622-629 (2012).

5. Volz, K. S. et al. Pericytes are progenitors for coronary artery smooth muscle. Elife 4, https://doi.org/10.7554/eLife.10036 (2015).

6. Zhou, B. et al. Epicardial progenitors contribute to the cardiomyocyte lineage in the developing heart. Nature 454, 109-113 (2008).

7. Trembley, M. A., Velasquez, L. S., de Mesy Bentley, K. L. \& Small, E. M. Myocardin-related transcription factors control the motility of epicardiumderived cells and the maturation of coronary vessels. Development 142, 21-30 (2015).

8. $\mathrm{Wu}, \mathrm{B}$. et al. Endocardial cells form the coronary arteries by angiogenesis through myocardial-endocardial VEGF signaling. Cell 151, 1083-1096 (2012).

9. $\mathrm{Su}, \mathrm{T}$. et al. Single-cell analysis of early progenitor cells that build coronary arteries. Nature 559, 356-362 (2018)

10. Lavine, K. J. et al. Endocardial and epicardial derived FGF signals regulate myocardial proliferation and differentiation in vivo. Dev. Cell 8, 85-95 (2005).

11. Cavallero, $\mathrm{S}$. et al. CXCL12 signaling is essential for maturation of the ventricular coronary endothelial plexus and establishment of functional coronary circulation. Dev. Cell 33, 469-477 (2015).

12. Lavine, K. J. et al. Fibroblast growth factor signals regulate a wave of Hedgehog activation that is essential for coronary vascular development. Genes Dev. 20, 1651-1666 (2006).

13. Cao, J. et al. Single epicardial cell transcriptome sequencing identifies Caveolin 1 as an essential factor in zebrafish heart regeneration. Development 143, 232-243 (2016).

14. Weinberger, M., Simoes, F. C., Patient, R., Sauka-Spengler, T. \& Riley, P. R. Functional heterogeneity within the developing zebrafish epicardium. Dev. Cell 52, 574-590.e576 (2020).

15. Lupu, I. E., Redpath, A. N. \& Smart, N. Spatiotemporal analysis reveals overlap of key proepicardial markers in the developing murine heart. Stem Cell Rep. 14, 770-787 (2020).

16. Bielle, F. et al. Slit2 activity in the migration of guidepost neurons shapes thalamic projections during development and evolution. Neuron 69, 1085-1098 (2011).

17. Han, X. et al. Mapping the mouse cell atlas by microwell-seq. Cell 173, 1307 (2018).

18. Bochmann, L. et al. Revealing new mouse epicardial cell markers through transcriptomics. PLoS ONE 5, e11429 (2010).

19. Wu, S. P., Dong, X. R., Regan, J. N., Su, C. \& Majesky, M. W. Tbx18 regulates development of the epicardium and coronary vessels. Dev. Biol. 383, 307-320 (2013).

20. Xiao, Y. et al. Hippo signaling plays an essential role in cell state transitions during cardiac fibroblast development. Dev. Cell 45, 153-169 e156 (2018).

21. Li, G. et al. Transcriptomic profiling maps anatomically patterned subpopulations among single embryonic cardiac cells. Dev. Cell 39, 491-507 (2016).

22. Karve, T. M. \& Rosen, E. M. B-cell translocation gene 2 (BTG2) stimulates cellular antioxidant defenses through the antioxidant transcription factor NFE2L2 in human mammary epithelial cells. J. Biol. Chem. 287, 31503-31514 (2012).

23. Lopez-Pajares, V. et al. A LncRNA-MAF:MAFB transcription factor network regulates epidermal differentiation. Dev. Cell 32, 693-706 (2015).

24. Lee, Y. J. et al. Soluble HSPB1 regulates VEGF-mediated angiogenesis through their direct interaction. Angiogenesis 15, 229-242 (2012).
25. Rodriguez, P. et al. The non-canonical NOTCH ligand DLK1 exhibits a novel vascular role as a strong inhibitor of angiogenesis. Cardiovasc. Res. 93 232-241 (2012)

26. Ichikawa-Shindo, Y. et al. The GPCR modulator protein RAMP2 is essential for angiogenesis and vascular integrity. J. Clin. Invest. 118, 29-39 (2008).

27. Courtwright, A. et al. Secreted frizzle-related protein 2 stimulates angiogenesis via a calcineurin/NFAT signaling pathway. Cancer Res. 69, 4621-4628 (2009).

28. Cacchiarelli, D. et al. Aligning single-cell developmental and reprogramming trajectories identifies molecular determinants of myogenic reprogramming outcome. Cell Syst. 7, 258-268 e253 (2018).

29. Trapnell, C. et al. The dynamics and regulators of cell fate decisions are revealed by pseudotemporal ordering of single cells. Nat. Biotechnol. 32, 381-386 (2014).

30. Mahtab, E. A. et al. Cardiac malformations and myocardial abnormalities in podoplanin knockout mouse embryos: correlation with abnormal epicardial development. Dev. Dyn. 237, 847-857 (2008).

31. Kang, J. et al. PDGF-A as an epicardial mitogen during heart development. Dev. Dyn. 237, 692-701 (2008).

32. Meadows, S. M. et al. Integration of repulsive guidance cues generates avascular zones that shape mammalian blood vessels. Circ. Res. 110, 34-46 (2012).

33. Landerholm, T. E. et al. A role for serum response factor in coronary smooth muscle differentiation from proepicardial cells. Development 126, 2053-2062 (1999).

34. Trembley, M. A., Velasquez, L. S. \& Small, E. M. Epicardial outgrowth culture assay and ex vivo assessment of epicardial-derived cell migration. J. Vis. Exp. https://doi.org/10.3791/53750 (2016).

35. Zhu, X. et al. Age-dependent fate and lineage restriction of single NG2 cells Development 138, 745-753 (2011).

36. Fang, J. S. et al. Shear-induced Notch-Cx37-p27 axis arrests endothelial cell cycle to enable arterial specification. Nat. Commun. 8, 2149 (2017).

37. Tian, X. et al. Subepicardial endothelial cells invade the embryonic ventricle wall to form coronary arteries. Cell Res. 23, 1075-1090 (2013).

38. Kalucka, J. et al. Single-cell transcriptome atlas of murine endothelial cells. Cell 180, 764-779 e720 (2020)

39. Zhang, Z. \& Zhou, B. Accelerated coronary angiogenesis by vegfr1-knockout endocardial cells. PLoS ONE 8, e70570 (2013).

40. Kidoya, H. et al. APJ regulates parallel alignment of arteries and veins in the skin. Dev. Cell 33, 247-259 (2015).

41. Saint-Geniez, M., Argence, C. B., Knibiehler, B. \& Audigier, Y. The msr/apj gene encoding the apelin receptor is an early and specific marker of the venous phenotype in the retinal vasculature. Gene Expr. Patterns 3, 467-472 (2003).

42. $\mathrm{Li}, \mathrm{S}$. et al. Slit2 promotes angiogenic activity via the Robo1-VEGFR2-ERK1/2 pathway in both in vivo and in vitro studies. Invest. Ophthalmol. Vis. Sci. 56, 5210-5217 (2015)

43. Jones, C. A. et al. Slit2-Robo4 signalling promotes vascular stability by blocking Arf6 activity. Nat. Cell Biol. 11, 1325-1331 (2009).

44. Franco, C. A. et al. Non-canonical Wnt signalling modulates the endothelia shear stress flow sensor in vascular remodelling. Elife 5, e07727 (2016).

45. Franco, C. A. et al. Dynamic endothelial cell rearrangements drive developmental vessel regression. PLoS Biol. 13, e1002125 (2015).

46. Acharya, A. et al. The bHLH transcription factor Tcf21 is required for lineagespecific EMT of cardiac fibroblast progenitors. Development 139, 2139-2149 (2012).

47. Braitsch, C. M., Combs, M. D., Quaggin, S. E. \& Yutzey, K. E. Pod1/Tcf21 is regulated by retinoic acid signaling and inhibits differentiation of epicardiumderived cells into smooth muscle in the developing heart. Dev. Biol. $\mathbf{3 6 8}$, 345-357 (2012)

48. Aghajanian, $\mathrm{H}$. et al. Coronary vasculature patterning requires a novel endothelial ErbB2 holoreceptor. Nat. Commun. 7, 12038 (2016).

49. Wu, C. C., Jeratsch, S., Graumann, J. \& Stainier, D. Y. R. Modulation of mammalian cardiomyocyte cytokinesis by the extracellular matrix. Circ. Res. 127, 896-907 (2020).

50. Mommersteeg, M. T. et al. Slit-roundabout signaling regulates the development of the cardiac systemic venous return and pericardium. Circ. Res. 112, 465-475 (2013).

51. Park, K. W. et al. Robo4 is a vascular-specific receptor that inhibits endothelial migration. Dev. Biol. 261, 251-267 (2003).

52. You, L. R. et al. Suppression of Notch signalling by the COUP-TFII transcription factor regulates vein identity. Nature 435, 98-104 (2005)

53. Pjanic, M. et al. Genetics and genomics of coronary artery disease. Curr. Cardiol. Rep. 18, 102 (2016).

54. Polizzotti, B. D. et al. Neuregulin stimulation of cardiomyocyte regeneration in mice and human myocardium reveals a therapeutic window. Sci. Transl. Med. 7, 281ra245 (2015).

55. White, I. A., Gordon, J., Balkan, W. \& Hare, J. M. Sympathetic reinnervation is required for mammalian cardiac regeneration. Circ. Res. 117, 990-994 (2015). 
56. Mahmoud, A. I. et al. Nerves regulate cardiomyocyte proliferation and heart regeneration. Dev. Cell 34, 387-399 (2015).

57. Li, Z. et al. Single-cell transcriptome analyses reveal novel targets modulating cardiac neovascularization by resident endothelial cells following myocardial infarction. Eur. Heart J. 40, 2507-2520 (2019).

58. Dube, K. N. et al. Recapitulation of developmental mechanisms to revascularize the ischemic heart. JCI Insight 2, https://doi.org/10.1172/jci. insight.96800 (2017).

59. Tang, J. et al. Genetic fate mapping defines the vascular potential of endocardial cells in the adult heart. Circ. Res. 122, 984-993 (2018).

60. Zhou, B. et al. Adult mouse epicardium modulates myocardial injury by secreting paracrine factors. J. Clin. Invest. 121, 1894-1904 (2011).

61. Duan, J. et al. Wnt1/betacatenin injury response activates the epicardium and cardiac fibroblasts to promote cardiac repair. EMBO J. 31, 429-442 (2012).

62. Miano, J. M. et al. Restricted inactivation of serum response factor to the cardiovascular system. Proc. Natl Acad. Sci. USA 101, 17132-17137 (2004).

63. Ramilowski, J. A. et al. A draft network of ligand-receptor-mediated multicellular signalling in human. Nat. Commun. 6, 7866 (2015).

64. Durinck, S., Spellman, P. T., Birney, E. \& Huber, W. Mapping identifiers for the integration of genomic datasets with the R/Bioconductor package biomaRt. Nat. Protoc. 4, 1184-1191 (2009).

65. Durinck, S. et al. BioMart and Bioconductor: a powerful link between biological databases and microarray data analysis. Bioinformatics 21, 3439-3440 (2005).

66. Wickham, H. et al. Welcome to the Tidyverse. J. Open Res. Softw. 4, 1686 (2019).

67. Hariharan, N. et al. Nucleostemin rejuvenates cardiac progenitor cells and antagonizes myocardial aging. J. Am. Coll. Cardiol. 65, 133-147 (2015).

68. Agullo-Pascual, E. et al. Super-resolution imaging reveals that loss of the Cterminus of connexin 43 limits microtubule plus-end capture and $\mathrm{NaV1.5}$ localization at the intercalated disc. Cardiovasc. Res. 104, 371-381 (2014).

69. van der Walt, S. et al. scikit-image: image processing in Python. PeerJ 2, e453 (2014).

70. Coelho, L. P. Mahotas: open source software for scriptable computer vision. J. Open Res. Softw. 1, e3 (2013).

\section{Acknowledgements}

We thank Bradford Berk and Charles Lowenstein for discussions; Matthew Cochran for assistance at the University of Rochester Medical Center Flow Cytometry Core; Michelle Zanche, Marlene Balys, and the University of Rochester Genomics Research Center for assistance with RNA-sequencing; Erika Flores Medina for assistance with histology; and Linda Callahan for assistance with microscopy. This work was supported by NIHT32HL066988, NIH-F32HL134206, and the American Heart Association 19CDA34590003 (to P.Q.); NIH-T32HL07572, NIH-T32HL066988, NIH-T32GM068411, the American Heart Association 16PRE3049000, and the Howard Hughes Medical Institute Med-IntoGrad program (to M.A.T); American Heart Association 20PRE35210597 and NIHF30HL150883 (to A.M.); an Excellence Scholarship for the Rafael del Pino Foundation (to M.P.-H.); NIH-R01-HL134328, NIH-R01-HL145911, NIH-R01-HL146514, and Fondation Leducq Transatlantic Network (to M.D.); NIH-R01-HL136179 (to E.M.S. and M.D.); NIH-R01HL133761, NIH-R01HL120919, NIH-R01HL144867, NIH-UL1-TR002001, and NYSTEM-C32566GG (to E.M.S.).

\section{Author contributions}

P.Q. designed the experiments, analyzed data, and wrote the manuscript with input from E.M.S.; P.Q. isolated tissues and cells for in vitro and ex vivo experiments, performed flow cytometry, RNA analyses, immunofluorescence, and FISH, with experimental assistance from A.M., M.A.T., R.A.D.J., and E.D.C.; J.A.M, J.R.M., and C.D.B. performed bioinformatic analyses, under the guidance of J.M.A.; M.P.-H. performed imaging analyses under the supervision of M.D. E.M.S. conceived of the study, supervised the research, and co-wrote the manuscript with input from all authors.

\section{Competing interests}

The authors declare no competing interests.

\section{Additional information}

Supplementary information The online version contains supplementary material available at https://doi.org/10.1038/s41467-021-24414-z.

Correspondence and requests for materials should be addressed to E.M.S.

Peer review information Nature Communications thanks Bin Zhou and the other anonymous reviewer(s) for their contribution to the peer review of this work.

Reprints and permission information is available at http://www.nature.com/reprints

Publisher's note Springer Nature remains neutral with regard to jurisdictional claims in published maps and institutional affiliations.

Open Access This article is licensed under a Creative Commons Attribution 4.0 International License, which permits use, sharing, adaptation, distribution and reproduction in any medium or format, as long as you give appropriate credit to the original author(s) and the source, provide a link to the Creative Commons license, and indicate if changes were made. The images or other third party material in this article are included in the article's Creative Commons license, unless indicated otherwise in a credit line to the material. If material is not included in the article's Creative Commons license and your intended use is not permitted by statutory regulation or exceeds the permitted use, you will need to obtain permission directly from the copyright holder. To view a copy of this license, visit http://creativecommons.org/ licenses/by/4.0/.

(C) The Author(s) 2021 